\title{
Phosphorylation-mediated PTEN conformational closure and deactivation revealed with protein semisynthesis
}

\author{
David Bolduc', Meghdad Rahdar², Becky Tu-Sekine ${ }^{3}$, Sindhu Carmen Sivakumaren', \\ Daniel Raben ${ }^{3}$, L Mario Amzel ${ }^{4}$, Peter Devreotes ${ }^{5}$, Sandra B Gabelli, ${ }^{4,6 *}$, Philip Cole ${ }^{1 *}$
}

${ }^{1}$ Department of Pharmacology and Molecular Sciences, Johns Hopkins University School of Medicine, Baltimore, United States; ${ }^{2}$ Department of Pharmacology, University of California, San Diego, San Diego, United States; ${ }^{3}$ Department of Biological Chemistry, Johns Hopkins University School of Medicine, Baltimore, United States; ${ }^{4}$ Department of Biophysics and Biophysical Chemistry, Johns Hopkins University School of Medicine, Baltimore, United States; ${ }^{5}$ Department of Cell Biology, Johns Hopkins University School of Medicine, Baltimore, United States; ${ }^{6}$ Departments of Medicine and Oncology, Johns Hopkins University School of Medicine, Baltimore, United States

*For correspondence: gabelli@ jhmi.edu (SBG); pcole@jhmi.edu (PC)

Competing interests: See page 16

Funding: See page 16

Received: 28 February 2013

Accepted: 07 June 2013

Published: 09 July 2013

Reviewing editor: Barbara Imperiali, Massachusetts Institute of Technology, United States

(c) Copyright Bolduc et al. This article is distributed under the terms of the Creative Commons Attribution License, which permits unrestricted use and redistribution provided that the original author and source are credited.
Abstract The tumor suppressor $\mathrm{PIP}_{3}$ phosphatase PTEN is phosphorylated on four clustered Ser/Thr on its C-terminal tail (aa 380-385) and these phosphorylations are proposed to induce a reduction in PTEN's plasma membrane recruitment. How these phosphorylations affect the structure and enzymatic function of PTEN is poorly understood. To gain insight into the mechanistic basis of PTEN regulation by phosphorylation, we generated semisynthetic site-specifically tetra-phosphorylated PTEN using expressed protein ligation. By employing a combination of biophysical and enzymatic approaches, we have found that purified tail-phosphorylated PTEN relative to its unphosphorylated counterpart shows reduced catalytic activity and membrane affinity and undergoes conformational compaction likely involving an intramolecular interaction between its C-tail and the C2 domain. Our results suggest that there is a competition between membrane phospholipids and PTEN phospho-tail for binding to the $\mathrm{C} 2$ domain. These findings reveal a key aspect of PTEN's regulation and suggest pharmacologic approaches for direct PTEN activation.

DOI: 10.7554/eLife.00691.001

\section{Introduction}

PTEN (phosphatase and tensin homolog deleted on chromosome 10) suppresses cell proliferation, migration and survival by dephosphorylating the lipid second messenger phosphatidyl inositol 3,4,5triphosphate $\left(\mathrm{PIP}_{3}\right)$, thereby opposing phosphatidyl inositol-3 kinase (PI3K) signaling and preventing AKT protein kinase activation (Maehama and Dixon, 1998; Myers et al., 1998; Sun et al., 1999; lijima and Devreotes, 2002). PTEN is one of the most frequently mutated genes in cancer, with inactivating mutations found in many solid tumor types (Li et al., 1997; Steck et al., 1997). The PTEN gene has been shown to be inactivated through somatic and germline mutations as well as transcriptionally suppressed through epigenetic mechanisms (Salvesen et al., 2001; Meng et al., 2007; Hollander et al., 2011). Homozygous deletion of PTEN is embryonically lethal in mice while heterozygous mice are predisposed to developing tumors (Suzuki et al., 1998; Di Cristofano et al., 1999; Podsypanina et al., 1999). Germline mutations in PTEN predispose people to spontaneous tumor formation as seen in Cowden syndrome (Nelen et al., 1997).

PTEN is a $47 \mathrm{kDa}$ (403 aa) protein composed of a 'dual-specificity' phosphatase domain, a C2 domain that mediates membrane association, and a 52 aa C-terminal tail (Figure 1A). An X-ray 
eLife digest PTEN is an enzyme that is found in almost every tissue in the body, and its job is to stop cells dividing. If it fails to perform this job, the uncontrolled proliferation of cells can lead to the growth of tumors. PTEN stops cells dividing by localizing at the plasma membrane of a cell and removing a phosphate group from a lipid called $\mathrm{PIP}_{3}$ : this sends a signal, via the PI3K pathway, that suppresses the replication and survival of cells.

Three regions of PTEN are thought to be central to its biological functions: one of these regions, the phosphatase domain, is directly responsible for removing a phosphate group from the lipid $\mathrm{PIP}_{3}$; a second region, called the C2 domain, is known to be critical for PTEN binding to the cell membrane; however, the role of third region, called the C-terminal domain, is poorly understood.

Many proteins are regulated by the addition and removal of phosphate groups, and PTEN is no exception. In particular, it seems as if the addition of phosphate groups to four amino acid residues in the C-terminal domain can switch off the activity of PTEN, but the details of this process have been elusive.

Now, Bolduc et al. have employed a variety of biochemical and biophysical techniques to explore this process, finding that the addition of the phosphate groups reduced PTEN's affinity for the plasma membrane. At the same time, interactions between the C-terminal and C2 domains of the PTEN cause the shape of the enzyme to change in a way that 'buries' the residues to which the phosphate groups have been added.

In addition to offering new insights into PTEN, the work of Bolduc et al. could help efforts to identify compounds with clinical anti-cancer potential.

DOI: 10.7554/eLife.00691.002

structure of PTEN, lacking the apparently flexible N-terminus (aa 1-13), internal D-loop (aa 286-309) and C-tail (aa 353-403), shows tight-knit interactions between the catalytic and C2 domains (Lee et al., 1999). The PTEN protein is believed to be regulated by a variety of mechanisms including posttranslational modifications, protein-protein interactions, and protein-lipid interactions (Campbell et al., 2003; lijima et al., 2004; Walker et al., 2004; Vazquez et al., 2006; Denning et al., 2007; Chagpar et al., 2009; Shenoy et al., 2012; Song et al., 2012). Previously mapped PTEN posttranslational modifications include phosphorylation (Vazquez et al., 2000; Torres and Pulido, 2001; Miller et al., 2002; Al-Kouri et al., 2005; Cordier et al., 2012), acetylation (Okumura et al., 2006), ubiquitylation (Wang et al., 2007), and sumoylation (Huang et al., 2012). Phosphorylation of a cluster of Ser/Thr residues (Ser380, Thr382, Thr383, and Ser385) on PTEN's C-terminal tail have received considerable attention in studies on PTEN regulation and appears to be a major modification (Wu et al., 2000; Vazquez et al., 2001; Odriozola et al., 2007; Rahdar et al., 2009). Reported to be catalyzed by the protein kinases CK2 and/or GSK3 (Torres and Pulido, 2001; Al-Khouri et al., 2005), this phospho-cluster is mutationally sensitive in cell assays, with replacements by Ala driving PTEN to the plasma membrane (Rahdar et al., 2009). While the molecular mechanism of how this is achieved is uncertain, altered protein-protein interactions (Wu et al., 2000; Vazquez et al., 2001; Sumitomo et al., 2004; Takahashi et al., 2006; van Diepen et al., 2009; Huang et al., 2012) as well as conformational changes (Odriozola et al., 2007; Rahdar et al., 2009) in PTEN have been suggested to be induced by phosphorylation. It has been unclear if phosphorylation of PTEN has a direct effect on membrane association or is indirectly mediated through other macromolecular interactions. Most PTEN phosphorylation analyses have relied on cellular transfection experiments in which replacement of the Ser and Thr residues with Ala have been indirect indicators of phosphorylation function. However, Ser and Thr have different properties from Ala and such mutations may be misleading in deducing the action of a phosphoSer/phosphoThr (Vazquez et al., 2000; Torres and Pulido, 2001; Al-Khouri et al., 2005). What has been lacking thus far is a biochemical analysis of purified phosphorylated PTEN in which the phosphorylation sites are well-defined in position and stoichiometry. Inherent challenges in obtaining this material include the difficulty in using kinases for introducing phosphates site-specifically into PTEN and the proposed potential for PTEN autodephosphorylation (Zhang et al., 2012). To circumvent these issues, we employ here expressed protein ligation, a method for protein semisynthesis (Schwarzer and Cole, 2005), for generating 380,382,383,385-tetraphosphorylatedPTEN (4p-PTEN). Expressed protein ligation involves the production of a recombinant protein carrying a $\mathrm{C}$-terminal thioester by exploiting the action of an intein, and its chemoselective ligation to an N-Cys 


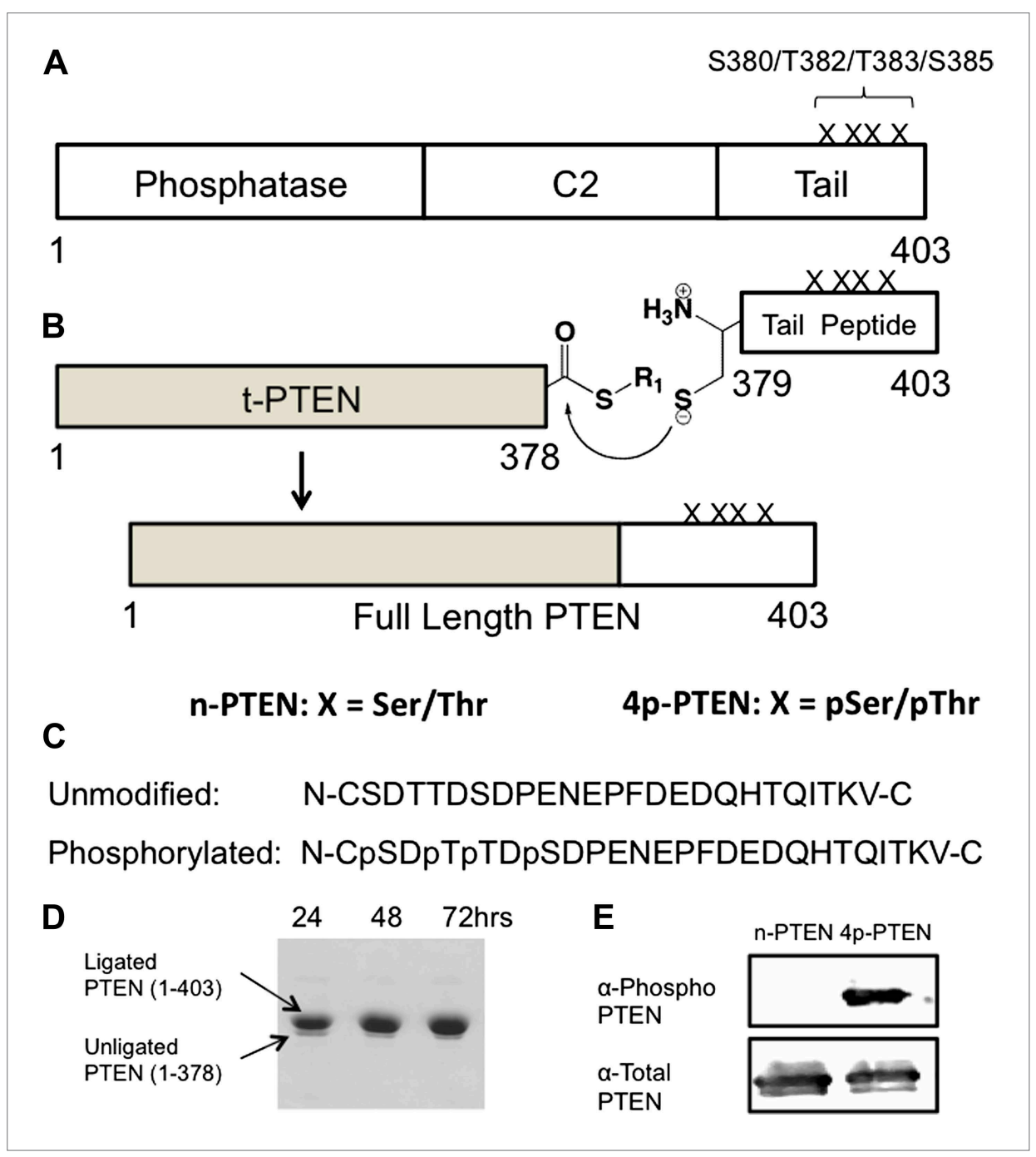

Figure 1. Generation of semisynthetic PTEN proteins. (A) PTEN is composed of phosphatase domain, a C2 domain, and a C-terminal tail that is phosphorylated multiple times within a cluster of Ser and Thr residues (S380/T382/T383/S385). (B) C-terminally truncated PTEN containing an intein generated thioester at its C-terminus is ligated to a synthetic PTEN tail peptide with or without phosphorylation at the S380/T382/T383/S385 cluster. The final product is full length PTEN in the phosphorylated (4p-PTEN) or unphosphorylated (n-PTEN) form. (C) C-terminal tail peptides were synthesized in the unphosphorylated form or phosphorylated at S380/T382/T383/S385. Note that the N-Cys replaces a natural Tyr in PTEN. (D) The ligation reaction precedes smoothly over $72 \mathrm{hr}$. (E) Western blot with an anti-phospho PTEN antibody reveals 4p-PTEN but not n-PTEN is phosphorylated. DOI: 10.7554/eLife.00691.003

The following figure supplements are available for figure 1:

Figure supplement 1 . Schematic views of $n-P T E N, 4 p-P T E N$ and t-PTEN

DOI: 10.7554/eLife.00691.004

Figure supplement 2. MALDI spectra for PTEN tail peptides and semisynthetic PTEN proteins. DOI: 10.7554/eLife.00691.005

Figure supplement 3. Size exclusion chromatography, Y379C enzymatic characterization and autophosphatase activity of PTEN

DOI: 10.7554/eLife.00691.006

containing synthetic peptide (Muir et al., 1998; Vila-Perelló and Muir, 2010). Within the synthetic peptide, phosphoSer/phosphoThr can be installed using standard chemical techniques at specific locations. We show here that, relative to the unphosphorylated form, $4 p-P T E N$ adopts a more compact conformation in which the $\mathrm{C}$-tail appears to interact with the $\mathrm{C} 2$ domain in a fashion that reduces its affinity for lipid membranes and diminishes its catalytic activity. 


\section{Results}

\section{Semisynthesis of tetraphosphorylated and unphosphorylated PTEN (4p-PTEN and n-PTEN)}

A requirement for expressed protein ligation is the correct positioning of a Cys for the native chemical ligation reaction (Muir et al., 1998; Schwarzer and Cole, 2005; Vila-Perelló and Muir, 2010). Initial attempts at generating semisynthetic PTEN focused on an Escherichia coli expression system. In this way, we showed that PTEN-intein fusion proteins allowed for generation of catalytically active recombinant PTEN thioester fragments (data not shown). We also determined using GSTPTEN that Cys was well-tolerated at the position needed for ligation, showing no change in catalytic activity induced by Y379C mutation (Figure 1-figure supplement 3D). However, expression in E. coli of the intein-fusion protein suffered from low yields of soluble PTEN protein expression $(<0.1 \mathrm{mg} / \mathrm{l}$ culture) which was insufficient for our needs.

We thus subcloned aa 1-378 of PTEN (t-PTEN) into a baculovirus plasmid for insect cell expression. Ligation with a tetraphosphorylated (and unphosphorylated as a control) N-Cys synthetic peptide aa 379-403 (Figure 1C) proceeded smoothly over $72 \mathrm{hr}$, providing 8-10 mg/l of culture purified semisynthteic PTEN protein (Figure 1B,D). Tetraphosphorylated (4p-PTEN), unphosphorylated (n-PTEN) and C-terminally truncated PTEN (t-PTEN) were generated in this way (Figure 1-figure supplement 1). Semisynthetic proteins were $>90 \%$ pure using Coomassie staining and their structural integrity confirmed by mass spectrometry (Figure 1-figure supplement 2C and D). Western blot with commercial anti-phospho-PTEN Ab showed that 4p-PTEN, and not n-PTEN, was appropriately phosphorylated (Figure 1E). It should also be noted that baculovirus systems have rarely been used for intein expression vectors (Pradhan et al., 1999), possibly in part because of the expression of chitinase in these hosts which interferes with the standard chitin affinity purification scheme. Nevertheless, we found the presence of chitinase to be a surmountable issue by a chromatographic pre-clearing step with a bed of fibrous cellulose. We also found that High Five insect cells rather than the more typical SF9 insect cells were critical for robust expression. Despite the theoretical concern about autodephosphorylation (Zhang et al., 2012), 4p-PTEN prepared in the presence of a PTEN phosphatase vanadate-based inhibitor was equally phosphorylated to that prepared in its absence (Figure 1-figure supplement 3E). Further experiments showed that 4p-PTEN did not undergo spontaneous pSer/pThr hydrolysis over $24 \mathrm{hr}$ as monitored by western blot (Figure 1-figure supplement 3F).

\section{Enzymatic characterization of 4p-PTEN}

Initial analysis of $4 p-P T E N$ catalytic activity was carried out with a soluble PIP $_{3}$ substrate containing hexanoyl rather than the more physiological palmitoyl chains using a phosphate release detection assay with malachite green (Van Veldhoven and Mannaert, 1987). These studies revealed a sixfold reduction in catalytic efficiency $\left(k_{\text {cat }} / K_{m}\right)$ conferred by $C$-terminal phosphorylation in which $4 p-P T E N$ shows a significantly higher soluble $\mathrm{PIP}_{3} \mathrm{~K}_{\mathrm{m}}$ (Figure 2A). To determine the enzymatic activity with a more physiologically relevant substrate, we analyzed 4p-PTEN's dephosphorylation of vesicle-incorporated $\mathrm{PIP}_{3}$ (containing palmitoyl chains) (McConnachie et al., 2003). 3'-[32P] PIP 3 was prepared by PI3-kinase and incorporated into vesicles containing unlabeled $\mathrm{PIP}_{3}$ and phosphatidylcholine (PC) (McConnachie et al., 2003). For interfacial enzymes such as PTEN, the bulk concentration as well as the surface concentration of the substrate in the lipid bilayer needs to be considered (Deems et al., 1975; Hendrickson and Dennis, 1984; McConnachie et al., 2003). We thus explored the rate of hydrolysis of $\mathrm{PIP}_{3}$ under conditions where the surface concentration of $\mathrm{PIP}_{3}$ was held constant while varying the bulk concentration of $\mathrm{PIP}_{3}$ and carrier lipid (phosphatidylcholine, $\mathrm{PC}$ ) proportionately (bulk dilution). In addition, we measured PTEN activity under conditions where the surface concentration of $\mathrm{PIP}_{3}$ was varied while its bulk concentration was held constant by varying the carrier lipid PC (surface dilution). As described in the 'Materials and methods' section, apparent $\mathrm{V}_{\max }$ and apparent $\mathrm{K}_{\mathrm{m}}$ values obtained from these experiments were fit to the equations in the 'Materials and methods' section associated with the equation below:

$$
\mathrm{V}_{0}=\left(\mathrm{V}_{\max }{ }^{*} \mathrm{X}_{\mathrm{s}}{ }^{*}\left[\mathrm{~S}_{0}\right]\right) /\left(\mathrm{i} K_{\mathrm{m}}{ }^{*} \mathrm{~K}_{\mathrm{s}}+\mathrm{i} K_{\mathrm{m}}{ }^{*}\left[\mathrm{~S}_{0}\right]+\mathrm{X}_{\mathrm{s}}{ }^{*}\left[\mathrm{~S}_{0}\right]\right)
$$

(McConnachie et al., 2003) in which $X_{S}$ is the surface concentration (mol fraction) of the substrate $\mathrm{PIP}_{3}, \mathrm{~S}_{0}$ is the bulk concentration of $\mathrm{PIP}_{3}, \mathrm{i} K_{\mathrm{m}}$ is the interfacial Michaelis constant (mol\%) and $\mathrm{K}_{\mathrm{s}}$ is the 

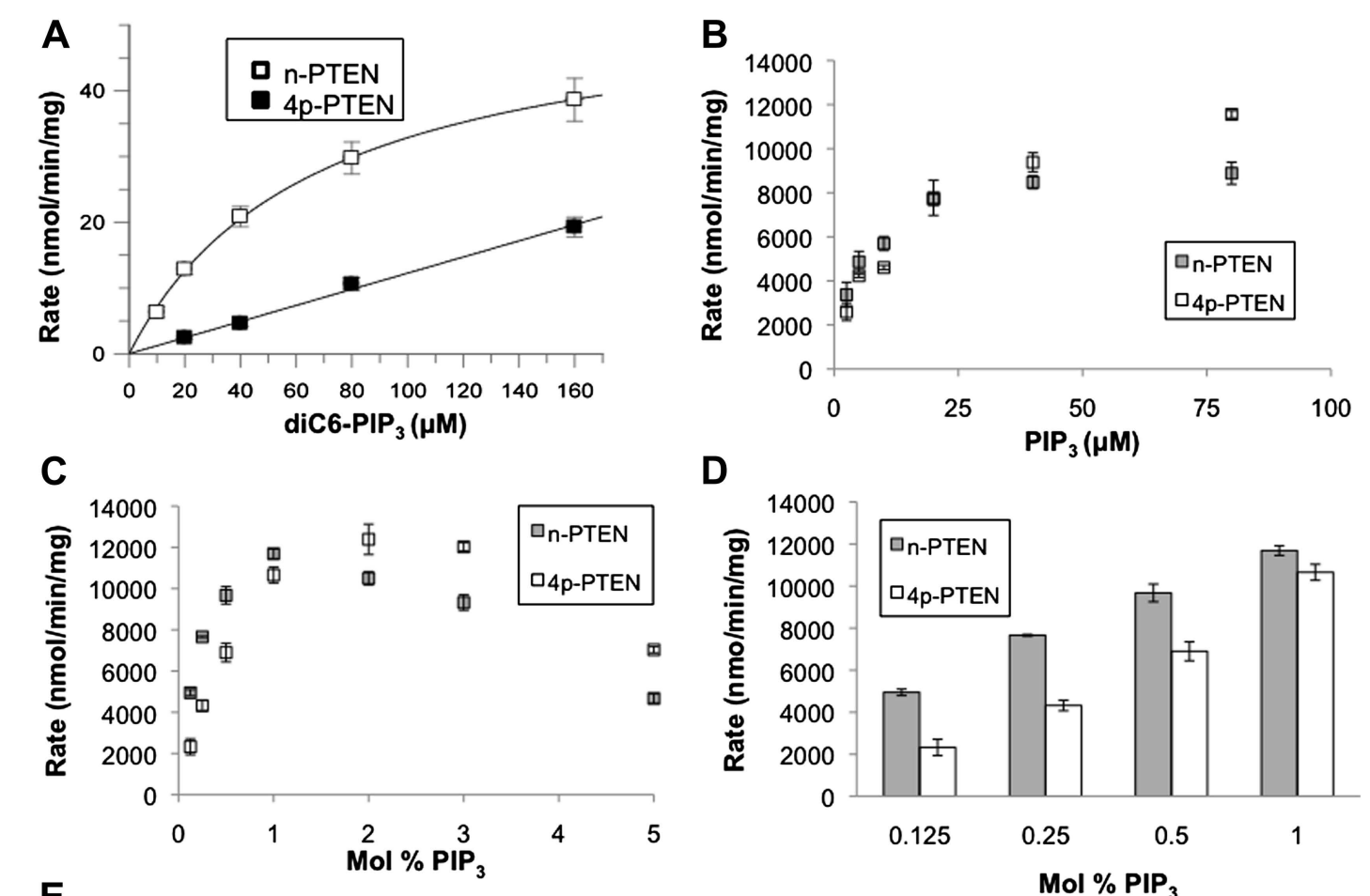

E

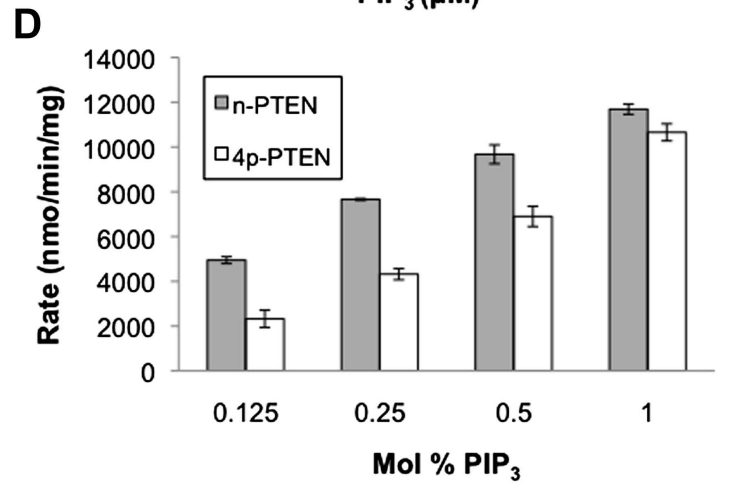

\begin{tabular}{|l|l|l|l|}
\hline & $i K_{m}(\mathrm{~mol} \%)$ & $\begin{array}{l}\mathrm{K}_{\mathrm{s}}(\mathrm{mM} \mathrm{Lipid}) \\
\left(1 \mathrm{~mol} \% \mathrm{PIP}_{3}\right)\end{array}$ & $\mathrm{k}_{\text {cat }}\left(\mathrm{s}^{-1}\right)$ \\
\hline n-PTEN & $0.52 \pm 0.04$ & $1.49 \pm 0.14$ & $11.22 \pm 0.3$ \\
\hline 4p-PTEN & $0.59 \pm 0.10$ & $4.06 \pm 0.75$ & $16.96 \pm 0.9$ \\
\hline
\end{tabular}

Figure 2. Soluble substrate activity and Interfacial kinetic analysis of semisynthetic PTEN. (A) PTEN activity to a soluble substrate, diC6-PIP 3 . (n-PTEN: $\left.\mathrm{k}_{\text {cat }}=2.6 \pm 0.1 \mathrm{~min}^{-1}, \mathrm{~K}_{\mathrm{m}}=67 \pm 4.2 \mu \mathrm{M}, \mathrm{k}_{\text {cat }} / \mathrm{K}_{\mathrm{m}}=0.038 \pm 0.001 \mathrm{~min}^{-1} \mu \mathrm{M}^{-1} ; 4 \mathrm{p}-\mathrm{PTEN}: \mathrm{k}_{\text {cat }} / \mathrm{K}_{\mathrm{m}}=0.005 \pm 0.0002 \mathrm{~min}^{-1} \mu \mathrm{M}^{-1}\right)(\mathrm{B}$ and C) PTEN activity to palmitoyl $\mathrm{PIP}_{3}$ incorporated into phosphatidylcholine vesicles. In the bulk dilution experiment (B) enzymatic activity for n-PTEN and 4p-PTEN was measured at a fixed surface concentration of $1 \% \mathrm{PIP}_{3}$ while the bulk concentration was varied. In the surface dilution experiment (C) activity was measured at a fixed bulk concentration of $50 \mu \mathrm{M} \mathrm{PIP}{ }_{3}$ while the surface concentration was varied. (D) 4p-PTEN has lower activity than n-PTEN only at low PIP 3 concentrations (E) Summary of the interfacial kinetic analysis of n-PTEN and 4p-PTEN. Data are reported as the mean \pm the SEM from three experiments performed in duplicate. Apparent $V_{\max }$ values were obtained from the best fit curves from the first four points of the surface dilution experiments. DOI: 10.7554/eLife.00691.007

The following figure supplements are available for figure 2:

Figure supplement 1. Interfacial kinetic analysis of semisynthetic PTENs.

DOI: 10.7554/eLife.00691.008

Figure supplement 2. Bulk and surface dilution curves of t-PTEN.

DOI: 10.7554/eLife.00691.009

Figure supplement 3. Anionic lipid stimulation of n-PTEN and 4p-PTEN.

DOI: 10.7554/eLife.00691.010

membrane dissociation constant for PTEN interaction with vesicles. Bulk dilution experiments yielded a rectangular hyperbola (Figure 2B), while surface dilution experiments showed apparent substrate inhibition at higher surface concentrations of $\mathrm{PIP}_{3}$ (Figure 2C). Using the lower substrate concentrations where substrate inhibition was minimal, the interfacial kinetic analysis revealed that $n-P T E N$ and $4 p-P T E N$ have similar $i K_{m}$ values with minor differences in $k_{c a t}$ values (Figure 2E and Figure 2-figure supplement $1 \mathrm{C}$ ). However, at $1 \% \mathrm{PIP}_{3}$, the $\mathrm{K}_{\mathrm{s}}$ value of $4 \mathrm{p}-\mathrm{PTEN}$ showed a $\sim$ threefold increase compared to that of $n$-PTEN, suggesting a decrease in binding affinity for the vesicle membrane 
when PTEN is phosphorylated (Figure 2E). n-PTEN $K_{s}$ and $i K_{m}$ values were similar to those of t-PTEN (Figure 2-figure supplement 2), indicating that the unphosphorylated tail extension does not hinder membrane interactions. Our data suggest that at low $(<1 \mathrm{~mol} \%) \mathrm{PIP}_{3}$ concentrations which are physiologic, the rate differential between n-PTEN and 4p-PTEN is significant (Figure 2D).

\section{Anionic lipid vesicle 4p-PTEN-interactions}

To further analyze the apparent membrane affinity loss conferred by PTEN tail phosphorylation, we explored the binding interactions of semisynthetic PTENs with vesicles containing anionic phospholipids. As reported previously for unphosphorylated PTEN produced in E. coli (McConnachie et al., 2003), we found that increasing levels of both phosphatidylserine (PS) and $\mathrm{PIP}_{2}$ in vesicles positively influence $\mathrm{PIP}_{3}$ dephosphorylation by both n-PTEN and 4p-PTEN (Figure 2-figure Supplement 3). We next carried out a series of PTEN-vesicle binding assays using PIP $_{2}$ and PS as membrane targeting lipids by measuring differential sedimentation fractionation. These experiments demonstrated markedly reduced membrane binding for $4 p$-PTEN vs n-PTEN under all conditions tested (Figure 3A,B and Figure 3-figure supplement 1). Both $n$-PTEN and 4p-PTEN show proportionally greater binding to vesicles as concentrations of $\mathrm{PIP}_{2}$ and PS are increased, but $4 p-P T E N$ compared with $n-P T E N$ requires a significantly higher concentration of anionic lipid to achieve a similar level of membrane sedimentation. For example, $7 \mathrm{~mol} \% \mathrm{PIP}_{2}$ sedimented about one-fifth of $4 \mathrm{p}-\mathrm{PTEN}$, which was comparable to the amount of $n$-PTEN sedimented by 3 mol\% PIP ${ }_{2}$ (Figure $3 A$ ). With 5 mol\% PS, the ratio of $n-P T E N: 4 p-P T E N$ sedimented was about 10:1 (Figure 3B). These results corroborate the PTEN affinity differences observed in the

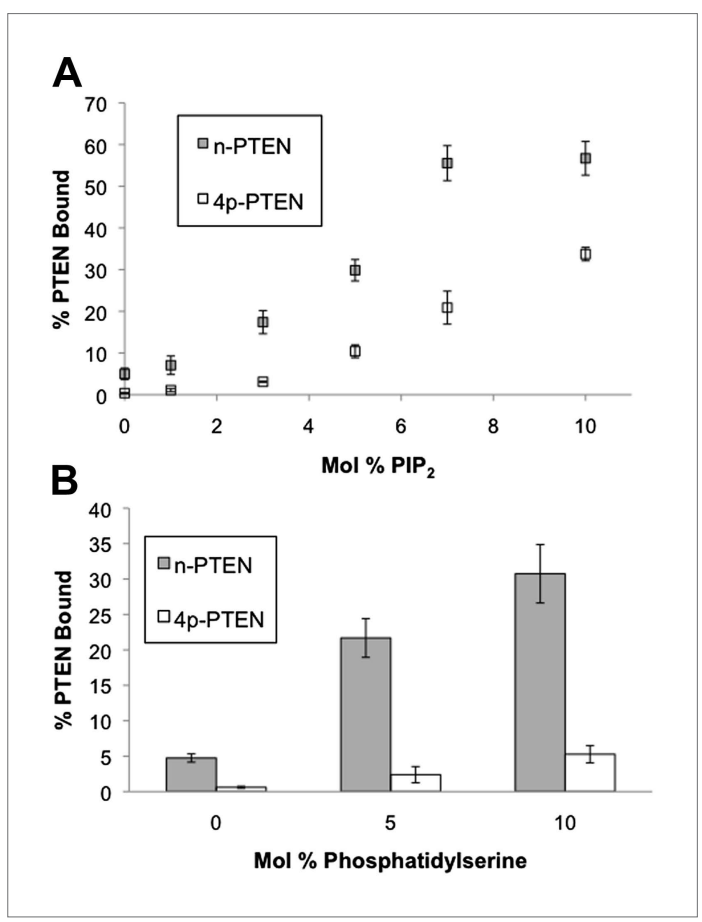

Figure 3. n-PTEN and 4p-PTEN binding to large multilamellar vesicles (LMVs). The percent of $n-P T E N$ and $4 p-P T E N$ bound to sedimented phosphatidylcholine LMVs incorporated with (A) $\mathrm{PIP}_{2}$ or $(\mathbf{B})$ phosphatidylserine was determined by quantification of western blot bands. Data are reported as the mean \pm the SEM of three separate experiments. DOI: 10.7554/eLife.00691.011

The following figure supplements are available for figure 3:

Figure supplement 1. n-PTEN and 4p-PTEN binding to large multilamellar vesicles (LMVs)

DOI: 10.7554/eLife.00691.012 phosphatase assays where C-tail phosphorylation of PTEN inhibited membrane binding.

\section{Conformational analysis of 4p-PTEN using ion exchange chromatography, limited proteolysis, and alkaline phosphatase sensitivity}

It has been proposed that C-terminal phosphorylation of PTEN may alter its conformation (Odriozola et al., 2007; Rahdar et al., 2009). In the course of purification of our semisynthetic PTENs by anion exchange chromatography, we made the paradoxical observation that the tetraphosphorylated PTEN reproducibly eluted at an earlier elution volume $(\sim 70 \mathrm{ml}$ and lower salt concentration, $90 \mathrm{mM} \mathrm{NaCl}$ ) and more sharply relative to the unphosphorylated protein $(\sim 100 \mathrm{ml}$, $140 \mathrm{mM} \mathrm{NaCl}$ ) (Figure 4A). Elution of $n-P T E N$ as one large peak followed by multiple small peaks was attributed to minor heterogeneous insect cell-mediated phosphorylation in the recombinant tail moiety on and near Thr366 and Ser370 as detected with a site-specific Ab (Figure 4figure supplement 1B). A similar elution pattern was observed with t-PTEN (Figure 4-figure supplement 1A). This heterogeneity is presumably collapsed under one peak in 4p-PTEN. Since $4 p$-PTEN has a nominal eight negative charge increase relative to n-PTEN, we were surprised that $4 \mathrm{p}$-PTEN showed decreased affinity to cationic resin. One explanation for this result is that, relative to n-PTEN, 4p-PTEN undergoes a conformational change which buries its negatively charged C-tail, reducing its availability for interacting with cationic resin. The fact that clusters of charges can be more important than 


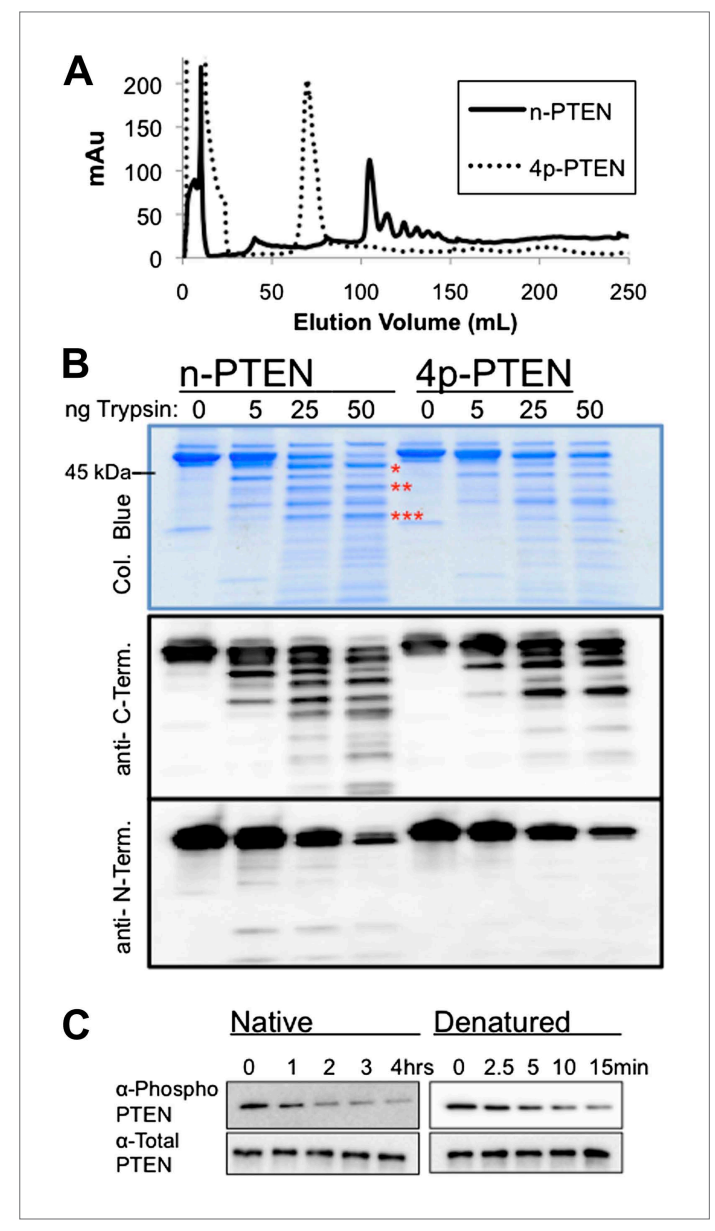

Figure 4. Conformational changes associated with PTEN phosphorylation. (A) With a gradient of $0-50 \% \mathrm{NaCl}$ over $250 \mathrm{ml}$ on an anion exchange column, 4p-PTEN elutes at $\sim 70 \mathrm{ml}$ while $\mathrm{n}$-PTEN elutes at $\sim 100 \mathrm{ml}$. (B) $2 \mu \mathrm{g}$ of $\mathrm{n}$-PTEN and $4 p$-PTEN were digested with varying amounts of trypsin then visualized by colloidal blue staining or by western blot with an antibody to the $\mathrm{N}$ - or $\mathrm{C}$-terminus of PTEN. Asterisks denote bands that are in higher abundance in the digestion of n-PTEN compared to $4 p-P T E N$. N-terminal sequencing of these bands identifies the cleavage sites as $\left(^{*}\right)$ R15, $\left(^{(*}\right)$ R84 and $\left(^{* * *}\right)$ R161. (C) Denatured $4 \mathrm{p}$-PTEN treated with $0.5 \mu \mathrm{M}$ alkaline phosphatase is significantly more sensitive to dephosphorylation of the tail phosphocluster compared to the native form of $4 \mathrm{p}$-PTEN treated with $1 \mu \mathrm{M}$ alkaline phosphatase. DOI: 10.7554/eLife.00691.013

The following figure supplements are available for figure 4:

Figure supplement 1. Non-tail cluster phosphorylation of PTEN expressed in High Five insect cells.

DOI: 10.7554/eLife.00691.014

Figure supplement 2. Native and denatured 4p-PTEN sensitivity to alkaline phosphatase.

DOI: 10.7554/eLife.00691.015 overall charge for protein-ion exchange resin interactions has been discussed previously (Chung et al., 1989; Hou et al., 2010).

To further explore the conformation of $4 p-P T E N$, we compared its susceptibility to trypsin proteolysis relative to $n$-PTEN. 4p-PTEN appeared more protease resistant vs its unphosphorylated counterpart as seen most clearly at 25 and $50 \mathrm{ng}$ trypsin (Figure 4B). Based on western blots with $\mathrm{N}$-terminal and $\mathrm{C}$-terminal PTEN Abs, several large metastable fragments still containing C-terminal epitopes were observed in n-PTEN, indicative of enhanced protease sensitivity in the catalytic domain in the unphosphorylated protein. $\mathrm{N}$-terminal sequencing identified several of these cleavage positions (Figure 4B).

Alkaline phosphatase sensitivity and western blotting was used to assess the exposure of the C-terminal 380-385 phospho cluster in $4 p-P T E N$ (Wang et al., 2002). The half-life of alkaline phosphatase-mediated dephosphorylation of $4 p-P T E N$ under the conditions used was found to be $\sim 60 \mathrm{~min}$ (Figure $4 \mathrm{C}$ and Figure 4figure supplement 2A). Serendipitously, we found that freeze-thawing dilute 4p-PTEN led to apparent denaturation of the protein since it was much more susceptible to alkaline phosphatase catalyzed dephosphorylation of the tail phosphate cluster. Under the same conditions, near complete dephosphorylation was observed within $7.5 \mathrm{~min}$. To more precisely determine this rate, we cut in half the concentration of alkaline phosphatase, and assumed that this process follows a pseudo-first-order kinetic mechanism. In this way, we estimate that native $4 p-P T E N$ vs denatured $4 p$-PTEN shows a 25 -fold reduced rate of alkaline phosphatase-catalyzed dephosphorylation of the tail phospho cluster (Figure $4 C$ and Figure 4-figure supplement 2B). We infer that this rate-differential suggests a closed:open $4 p-P T E N$ conformational equilibrium of $25: 1$ under the conditions of this experiment.

\section{Small angle X-ray scattering (SAXS) analysis of semisynthetic PTENs}

To gain more information on the structural changes induced by PTEN tail phosphorylation, we performed small angle X-ray scattering (SAXS) analysis of t-PTEN, n-PTEN, and 4p-PTEN. The scattering plots are shown in Figure 5A for each protein. The shape of the pair distance distribution function $p(r)$ plot for $n$-PTEN reveals a single hump with a large shoulder region at higher $r$ values

(Figure 5B). The shoulder region is indicative of a protein with an elongated shape (Putnam et al., 2007; Jacques and Trewhella, 2012), possibly due to the presence of the extended tail. The shoulder is reduced for t-PTEN and 4p-PTEN, suggesting the tail is no longer in an extended position (Figure 5B). 


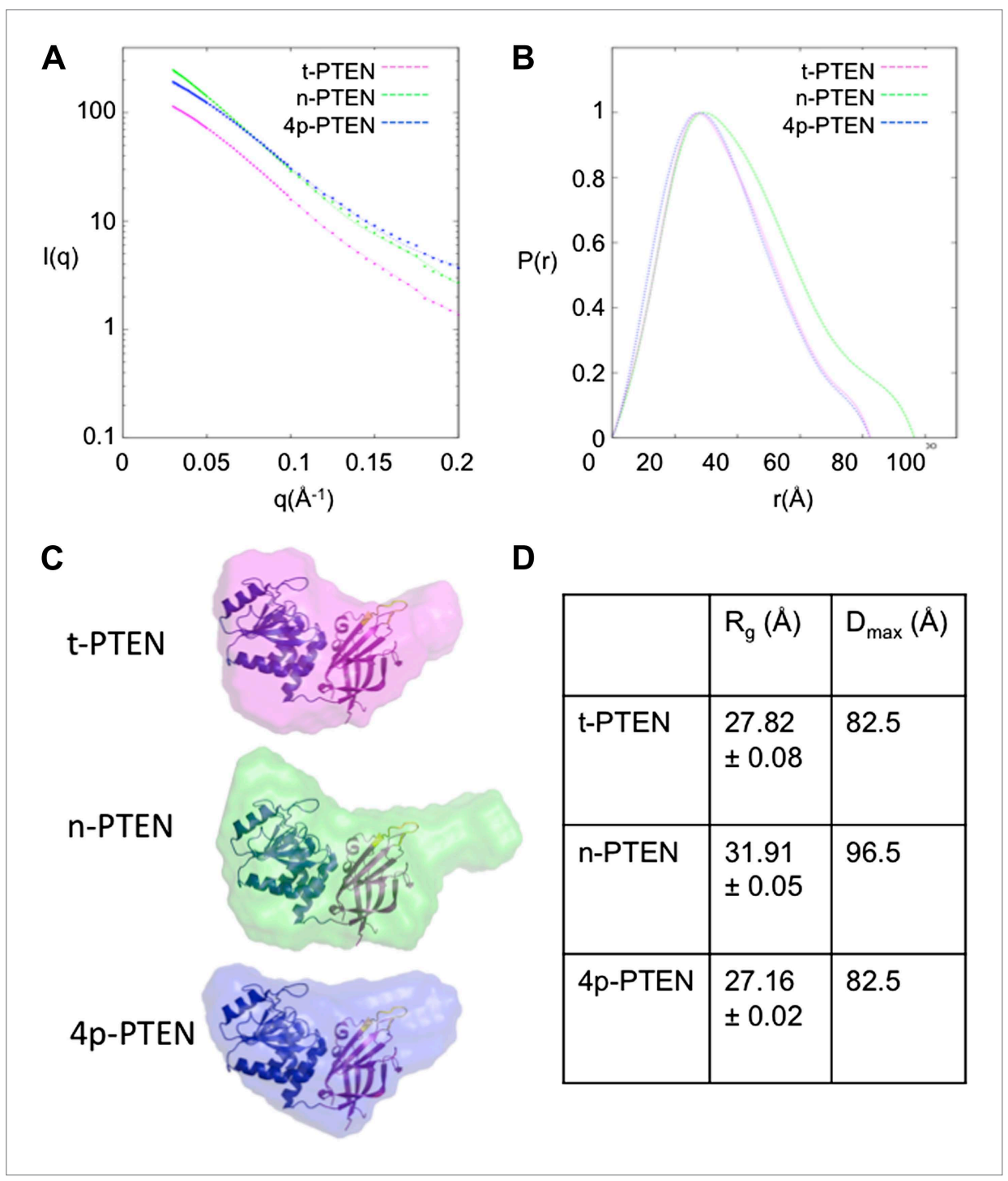

Figure 5. SAXS analysis for t-PTEN, n-PTEN and 4p-PTEN. (A) Scattering diagrams and (B) pair distribution function (Pofr) plots for t-PTEN, n-PTEN, and 4p-PTEN. (C) The molecular envelopes for t-PTEN, n-PTEN, and 4p-PTEN overlaid with the tailless crystal structure containing the phosphatase domain (blue), C2 domain (magenta), and CBRIII loop (yellow). (D) Summary of $R_{g}$ and $D_{\max }$ values for t-PTEN, n-PTEN, and 4p-PTEN.

DOI: 10.7554/eLife.00691.016

The following figure supplements are available for figure 5:

Figure supplement 1. Molecular envelopes of t-PTEN, n-PTEN and 4p-PTEN obtained from SAXS analysis. DOI: 10.7554/eLife.00691.017

The radius of gyration $\left(R_{\mathrm{g}}\right)$ and maximum dimension $\left(D_{\max }\right)$ of the PTEN particles can be used as measurements of protein size. $R_{g}$ values calculated from the Guinier and $p(r)$ plots are in agreement for each respective protein. Obtained from the $p(r)$ plot, the $R_{g}$ for $n$-PTEN $(31.91+/-0.054 \AA)$ is larger than it is for 4p-PTEN $(27.16+/-0.019 \AA)$ and t-PTEN $(27.82+/-0.083 \AA)$. $D_{\max }$ is also larger for $n-P T E N$ $(96.5 \AA)$ than it is for $4 p-P T E N(82.5 \AA)$ and t-PTEN (82.5 $\AA$ ) (Figure 5D).

Molecular envelopes of t-PTEN, n-PTEN and 4p-PTEN were generated from the scattering data using the ab initio modeling program DAMMIN (Putnam et al., 2007; Franke and Svergun, 2009; Jacques and Trewhella, 2012). Outputs from 10 DAMMIN runs, averaged for each protein using DAMAVER, are shown overlaid with the tailless crystal structure in Figure 5C (Figure 5-figure Supplement 1). The tailless crystal structure containing the phosphatase and C2 domains fits nicely into the two-lobed globular portion of each envelope. The molecular envelope for $n-P T E N$ 
reveals an elongated extension proximal to the C2 domain (Figure 5C). The molecular envelope for t-PTEN, which contains half of the 52 residue tail, shows a small extension, similar in length to that of $4 p-P T E N$, but perhaps not as wide. Interestingly, the 4p-PTEN phosphatase domain appears to undergo a modest change in shape relative to t-PTEN and n-PTEN (Figure 5C and Figure 5figure supplement 1 ).

\section{Mutagenesis analysis of semisynthetic PTENs}

Based on prior models as well as the proteolysis and SAXS experiments, we constructed several mutant semisynthetic PTENs to investigate possible PTEN residues that contribute to the closed conformation of 4p-PTEN (Figure 6-figure supplement 1). Semisynthetic phospho- and unphosphorylated PTEN containing four mutations K13A, R14A, R15A, and R161A ('N-mutant') was constructed to test the possibility that these basic residues in the $\mathrm{N}$-terminus, co-localized in the crystal structure and implicated in phospholipid binding, might be critical in stabilizing the closed $4 p-P T E N$ conformation. We also generated the penta-mutant unphosphorylated and phosphorylated PTENs containing K260A/K263A/K266A/K267A/K269A ('A5-mutant') and K260D/K263D/K266D/ K267D/K269D ('D5-mutant') as neutral and charge inverted forms that probe the importance of the CBRIII loop in the C2 domain to conformational constriction. A fourth construct of semisynthetic PTEN that included deletions in the N-terminus (aa 1-6), D-loop (aa 286-309), and C-terminus (aa 395-403, and also contained T366A/S370A), ('X-mutant') was prepared to facilitate comparisons to the crystallized form of PTEN which possessed the same N-terminal and 'D-loop' deletions (Lee et al., 1999). These semisynthetic PTEN mutants were generated analogously to that of n-PTEN and 4p-PTEN. Anion exchange chromatography showed the same paradoxical behavior for the phosphorylated forms of the N-mutant, A5-mutant, and X-mutant PTENs with faster than expected elution of these semisynthetic proteins. Of note, elution of the unligated $X$-mutant PTEN on anion exchange chromatography sharpened to a single peak compared to the broader distribution of unligated wt PTEN (t-PTEN), presumably because mutation of Thr366/ Ser370 abolishes the phosphorylation events that lead to heterogeneity (Figure 6-figure supplement 2). Moreover, N-mutant, A5-mutant, and X-mutant 4p-PTEN proteins showed nearly identical rates of dephosphorylation by alkaline phosphatase relative to wt $4 \mathrm{p}-\mathrm{PTEN}$ indicating that they are each in the same $\sim 25: 1$ equilibrium favoring the closed conformation (Figure 6A and Figure 6-figure supplement 3 ).

In contrast, the D5 mutant 4p-PTEN showed a distinctive biochemical behavior. With respect to anion exchange chromatography, D5 mutant 4p-PTEN eluted broadly with much of the protein eluting at higher $\mathrm{NaCl}$ concentrations than the unphosphorylated D5 mutant (Figure 6-figure supplement 4). Later elution is the expected profile for a typical phosphorylated protein relative to an unphosphorylated protein compared with the paradoxical pattern for $n$ - and $4 p$ - semisynthetic PTENs. In addition, D5 mutant 4p-PTEN was dephosphorylated about eightfold faster than the native wt 4p-PTEN and only threefold slower than denatured wt 4p-PTEN, implying that the tail phosphate cluster is dramatically more exposed in the D5 mutant (Figure 6A and Figure 6-figure Supplement 3). We considered the possibility that Asp substitutions of the Lys residues in the D5 mutant led to overall protein destabilization and denaturation. However, D5- mutant 4p-PTEN readily processed soluble $\mathrm{PIP}_{3}$ substrate, threefold faster than wt and A5-mutant 4p-PTENs (Figure 6B). Moreover, the D5-mutant 4p-PTEN phosphatase activity showed saturation with soluble $\mathrm{PIP}_{3}$ (Figure $6 \mathrm{C}$ ), with catalytic parameters approaching those of n-PTEN, consistent with a more open conformation than that of wt 4p-PTEN. Taken together, these results make clear that the penta-Asp substitutions in the CBRIII loop are non-denaturing but can significantly disrupt the closed conformation of $4 p-P T E N$, presumably by electrostatic repulsion of the anionic tail. As reported previously (Campbell et al., 2003), the activity of the N-mutant which contains mutations of K13, R14, and R15 to alanine, is reduced compared to wt (Figure 6B and Figure 6-figure supplement 5).

\section{Intermolecular interactions between t-PTEN and C-tail phosphopeptide}

We investigated the possibility that the synthetic C-tail tetraphosphorylated peptide (aa 379-403, $4 p-25 m e r)$ used in ligation could modulate the activity of truncated (t-PTEN, aa 1-378) in an intermolecular fashion using the soluble $\mathrm{PIP}_{3}$ substrate phosphatase assay. In this assay, t-PTEN showed similar behavior to n-PTEN, with saturable kinetics as a function of soluble $\mathrm{PIP}_{3}$ (compare Figures $7 \mathrm{~B}$ and $2 \mathrm{~A}$ ). 


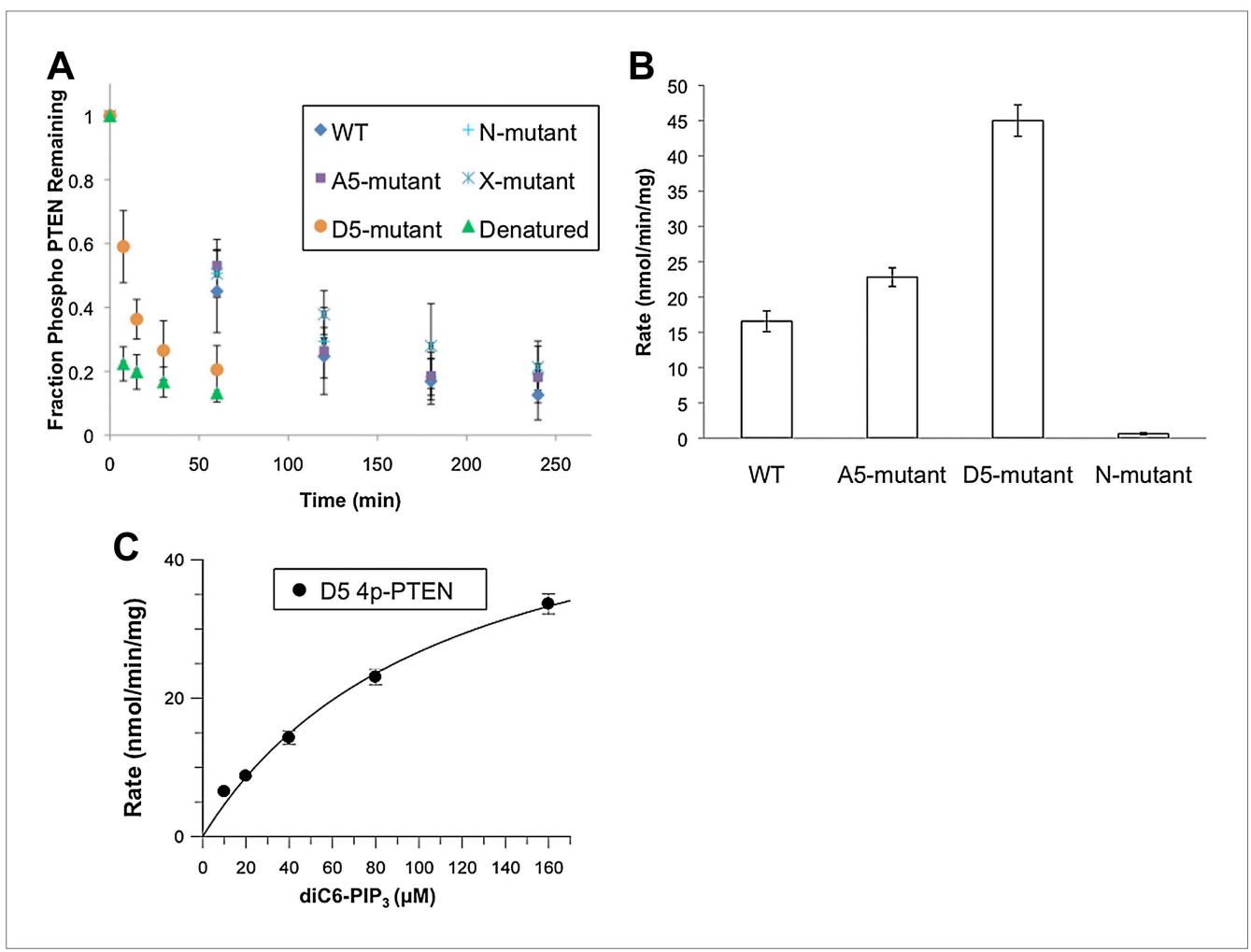

Figure 6. Phosphatase sensitivity and activity of 4p-PTEN and its mutants. (A) The rate of dephosphorylation of $4 p-P T E N$ and its mutants was measured by quantification of bands from western blot analysis after treatment of the PTEN protein with $1 \mu \mathrm{M}$ alkaline phosphatase. Data points are shown as the mean \pm the SEM of three experiments. (B) PTEN activity was measured against $160 \mu \mathrm{M}$ diC6-PIP 3 substrate. (C) $K_{m}$ curve of D5 4p-PTEN mutant $\left(k_{\text {cat }}=3.0 \pm 0.3 \mathrm{~min}^{-1}, \mathrm{~K}_{\mathrm{m}}=112 \pm 22 \mu \mathrm{M}, \mathrm{k}_{\text {cat }} / \mathrm{K}_{\mathrm{m}}=0.027 \pm 0.003 \mathrm{~min}^{-1} \mu \mathrm{M}^{-1}\right)$. Data points are shown as the mean \pm the SEM of three experiments performed in duplicate.

DOI: 10.7554/eLife.00691.018

The following figure supplements are available for figure 6:

Figure supplement 1. Schematic view of semisynthetic PTEN mutants. DOI: 10.7554/eLife.00691.019

Figure supplement 2. Anion exchange elution pattern of the PTEN X-mutant. DOI: 10.7554/eLife.00691.020

Figure supplement 3. Alkaline phosphatase sensitivity of $4 p-P T E N$ and mutant forms. DOI: 10.7554/eLife.00691.021

Figure supplement 4. Anion exchange chromatography elution profiles of phosphorylated and unphosphorylated D5 PTEN.

DOI: 10.7554/eLife.00691.022

Figure supplement 5. PTEN activity to diC6 PIP3.

DOI: 10.7554/eLife.00691.023

Of note, the C-tail phosphopeptide $4 \mathrm{p}-25 \mathrm{mer}$, but not the unphosphorylated peptide $\mathrm{n}-25 \mathrm{mer}$, was a potent inhibitor of t-PTEN soluble $\mathrm{PIP}_{3}$ phosphatase activity with an IC $\mathrm{C}_{50} \sim 1 \mu \mathrm{M}$ (Figure 7A). In the presence of $1 \mu \mathrm{M} 4 \mathrm{p}-25 \mathrm{mer}$, the t-PTEN phosphatase activity showed an increase in $K_{m}$ for soluble $\mathrm{PIP}_{3}$ (Figure 7B), mimicking the behavior of 4p-PTEN. Strikingly, the D5 mutant form of t-PTEN was resistant to the inhibition by $4 \mathrm{p}-25$ mer at concentrations up to $10 \mu \mathrm{M}$ of the peptide (Figure 7C). Analogous to its effects on t-PTEN phosphatase activity, $4 \mathrm{p}-25 \mathrm{mer}$ but not $\mathrm{n}-25 \mathrm{mer}$ inhibited t-PTEN binding to $\mathrm{PIP}_{2}$ vesicles, although less potently presumably because of competition for binding surfaces on the body of PTEN between the vesicle lipids and the $4 p-25$ mer tail peptide (Figure 7D). Taken together, these experiments reveal that the intermolecular effects of the C-tail phosphopeptide on the PTEN body resemble those proposed for the intramolecular conformational change in $4 p$-PTEN. 


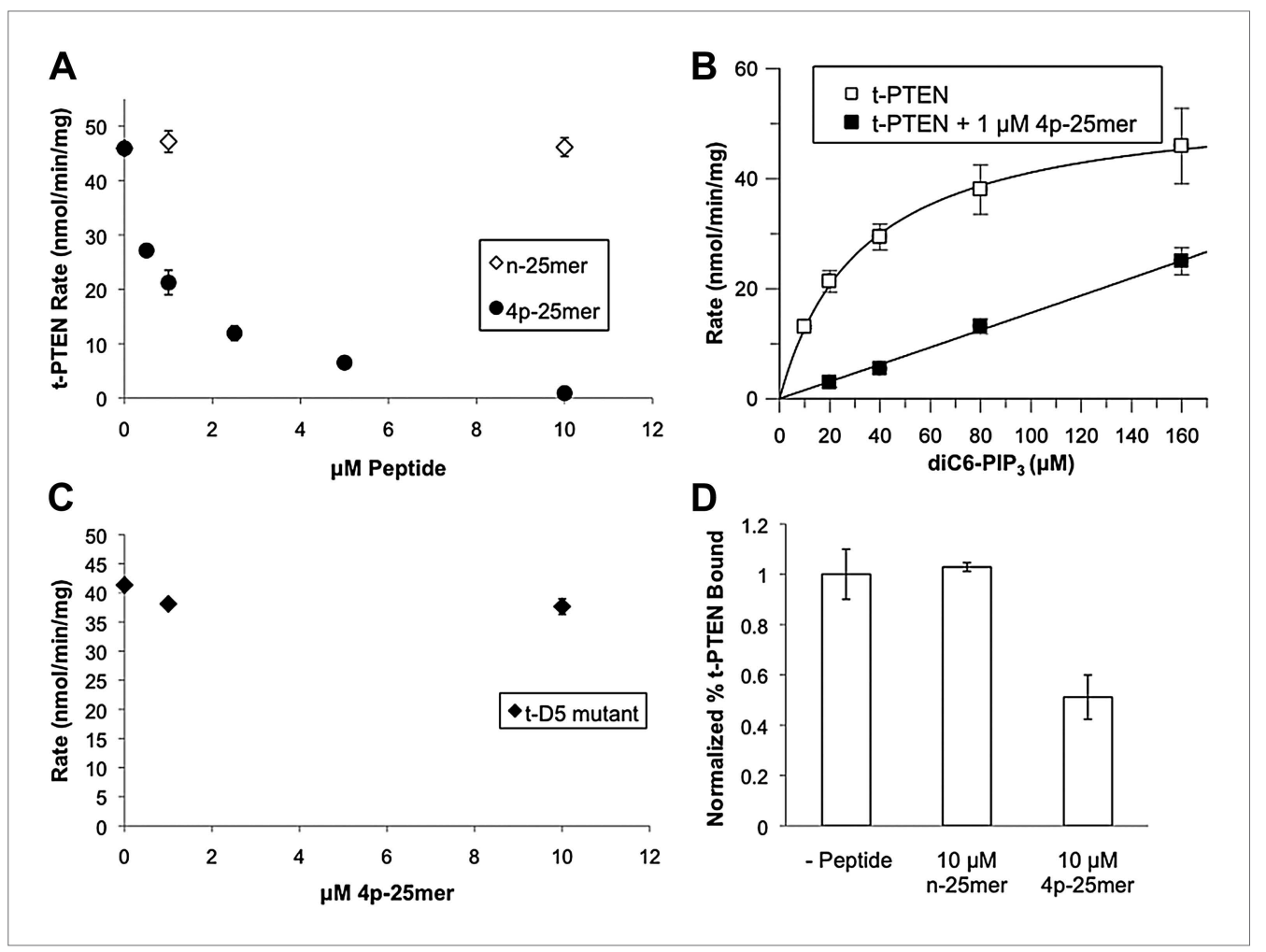

Figure 7. In trans peptide inhibition and binding of PTEN. (A) Tail peptide inhibition of t-PTEN with either $n-25$ mer or $4 \mathrm{p}-25 \mathrm{mer}$. (B) $\mathrm{K}_{\mathrm{m}}$ curve of t-PTEN in the absence $\left(\mathrm{k}_{\text {cat }}=2.9 \pm 0.1 \mathrm{~min}^{-1} ; \mathrm{K}_{\mathrm{m}}=33 \pm 2.1 \mu \mathrm{M}, \mathrm{k}_{\text {cat }} / \mathrm{K}_{\mathrm{m}}=0.088 \pm 0.004 \mathrm{~min}^{-1} \mu \mathrm{M}^{-1}\right)$ or presence $\left(k_{\text {cat }} / K_{m}=0.006 \pm 0.0003 \mathrm{~min}^{-1} \mu \mathrm{M}^{-1}\right)$ of $1 \mu \mathrm{M} 4 \mathrm{p}-25$ mer phosphopeptide. (C) Reduced inhibition of D5 t-PTEN mutant in the presence of $4 p-25$ mer peptide. (D) Vesicle sedimentation of t-PTEN in absence and presence of $10 \mu \mathrm{M}$ tail peptides. Data points are shown as the mean \pm the SEM of three experiments performed in duplicate. DOI: 10.7554/eLife.00691.024

\section{Discussion}

The evidence developed here points to a straightforward model for how phosphorylation regulates PTEN structure and function. Upon phosphorylation on the 380-385 Ser/Thr cluster, the PTEN C-terminal modified tail clamps down intramolecularly on the C2 domain in the vicinity of the CBRIII loop, preventing PTEN from binding the plasma membrane and reducing its catalytic action toward PIP $\mathrm{P}_{3}$ (Figure 8). The combined structural data including ion exchange chromatographic behavior, trypsin protease susceptibility, alkaline phosphatase sensitivity, and SAXS analysis point to a more compact $4 p-P T E N$ relative to $n-P T E N$ in which the phosphates on the tail are concealed.

While it is formally possible that the phospho-tail of PTEN could cause indirect effects on the C2 domain by binding elsewhere on the PTEN body, the accumulated evidence argues for a direct phospho-tail-C2 interaction. The SAXS results suggest that phospho-tail is in close proximity to the C2 domain. Replacement of the PTEN Lys cluster of the CBRIII loop with Asp residues (5D mutant) but not Ala residues (5A mutant) in 4p-PTEN renders the phospho-tail more susceptible to alkaline phosphatase hydrolysis, presumably because the anionic phosphate tail clashes with the Asp carboxylate negative charges. The intermolecular effects of the $4 \mathrm{p}-25 \mathrm{mer} C$-tail on the t-PTEN body and their reduced sensitivity conferred by CBRIII loop mutation further corroborate the phospho-tail-C2 interaction. Prior studies suggest that the five clustered Lys residues of the CBRIII loop are found on the membrane binding surface of the C2 domain, and their mutation decreases PTEN binding to lipid vesicles in vitro and plasma membranes in cell transfection experiments (Lee et al., 1999; McConnachie et al., 2003).

It is within the realm of possibility that 4 p-PTEN can bind to membrane in its closed conformation, albeit with significantly reduced affinity. Our best evidence against the possibility that conformationally closed $4 p$-PTEN binds membrane is that the interfacial $K_{m} s\left(i K_{m} s\right)$ for $4 p-P T E N, n-P T E N$, and t-PTEN are all the same, within error, in dephosphorylating vesicle-bound $\mathrm{PIP}_{3}$ (Figure 2). Since the $\mathrm{K}_{\mathrm{m}} \mathrm{s}$ for 


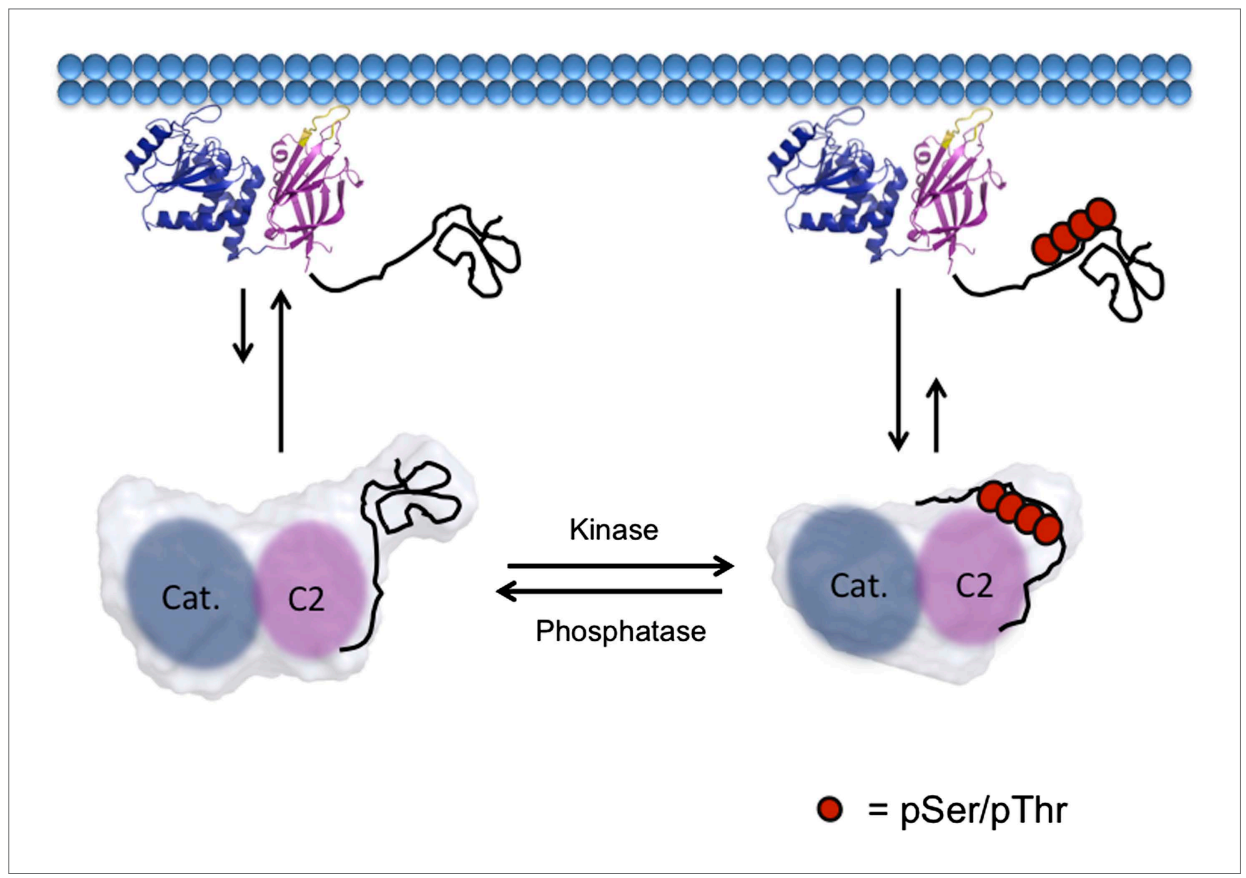

Figure 8. Model of PTEN regulation by phosphorylation. Upon phosphorylation, PTEN adopts a more compact conformation with the phosphorylated tail condensing around the CBRIII loop and membrane binding surface of the C2 domain, preventing it from binding to the plasma membrane. When dephosphorylated, the tail of PTEN is no longer bound tightly to the C2 domain, allowing for the open PTEN protein to bind efficiently to the plasma membrane. Both phosphorylated and unphosphorylated PTEN are in the same open conformation when bound to the plasma membrane.

DOI: 10.7554/eLife.00691.025

soluble $\mathrm{PIP}_{3}$ dephosphorylation are different for $4 \mathrm{p}$-PTEN and n-PTEN (Figure 2), it would be surprising that the $i K_{m} s$ would be the same if the conformation of $4 p-P T E N$ were closed when bound to vesicle. It is plausible, however, that some closed 4 p-PTEN with reduced membrane affinity is interacting with vesicle while in a catalytically impaired state, which would contribute only in a minor way to the $\mathrm{i}_{\mathrm{m}}$. Nevertheless, we also know how important the CBRIII loop is for membrane binding which, as discussed above, is likely abutting the phospho-tail in the closed conformation. Thus, the information available strongly supports the model in Figure 8.

We observed here that C-terminal phosphorylation of PTEN significantly reduces the enzyme's lipid bilayer affinity and also its catalytic efficiency with soluble $\mathrm{PIP}_{3}$ substrate and at low $(<1 \mathrm{~mol} \%) \mathrm{PIP}_{3}$ surface concentrations in vesicles. Since physiologic membrane surface concentrations of $\mathrm{PIP}_{3}$ are in the range of 0.001 mol\% or less, C-terminal phosphorylation of PTEN should confer an important reduction in enzymatic activity in vivo (Rahdar et alo, 2009). It is interesting that the soluble $\mathrm{PIP}_{3} \mathrm{~K}_{\mathrm{m}}$ for $4 p-P T E N$ vs n-PTEN is elevated whereas the interfacial $K_{m}\left(i_{m}\right)$ with vesicles containing $\mathrm{PIP}_{3}$ is the same for both enzyme forms. Both the trypsin protease pattern and SAXS analysis of 4p-PTEN vs n-PTEN reveal apparent structural/dynamic changes within the catalytic domain upon phosphorylation that may account for the altered phosphatase properties. Such conformational changes in the catalytic domain upon phosphorylation may come from direct interactions with the tail, though they could also be transmitted allosterically through tail- $\mathrm{C} 2$ binding since the $\mathrm{C} 2$ domain makes intimate contacts with the catalytic domain. It should be noted that the CBRIII loop, while critical for vesicle interaction, is dispensable for soluble $\mathrm{PIP}_{3}$ substrate processing, as shown here and previously (McConnachie et al., 2003). Thus, there are at least some differences between interactions of PTEN with soluble $\mathrm{PIP}_{3}$ and membrane-embedded $\mathrm{PIP}_{3}$.

Results from previous experiments involving co-immunoprecipitation of the co-transfected PTEN tail and body as separate pieces hinted at the potential of a conformational change induced by phosphorylation (Rahdar et al., 2009). However, in contrast to the findings here, the co-immunoprecipitation of the PTEN tail and body was disrupted by the altered residues in the $\mathrm{N}$-mutant and A5-mutant 
PTENs. Since the N-mutant and A5-mutant 4p-PTENs showed the same apparent closed equilibrium constant as wt 4p-PTEN, this suggests that there may be differences in this trans interaction identified in mammalian cell extracts vs the closed conformation of the intact 4p-PTEN molecule analyzed in the current study. Indeed, we found higher than expected apparent affinity between the $4 p-25 \mathrm{mer}$ and the t-PTEN body $\left(I_{50} 1 \mu \mathrm{M}\right)$ and more complete inhibition (>95\%) of the lipid phosphatase activity of t-PTEN by the $4 p-25$ mer with soluble $\mathrm{PIP}_{3}$ substrate than would be predicted based on results with $4 p$-PTEN (4p-PTEN:n-PTEN activity was $\sim 6: 1)$. The $~ 25: 1$ equilibrium favoring the closed conformation of $4 p$-PTEN based on alkaline phosphatase susceptibility seems relatively small compared with an apparent $K_{d}$ of $\sim 1 \mu \mathrm{M}$ for the $4 p-25$ mer-t-PTEN intermolecular interaction. Such results may suggest structural differences in the intermolecular complex vs the intramolecular conformational change. While there are several plausible explanations for this, one interesting possibility is that there may be energetic strain associated with achieving conformational closure in the intramolecular case of $4 p-P T E N$ not associated with a phospho-tail-t-PTEN intermolecular interaction.

Long-range intramolecular protein conformational switching induced by phosphorylation has been observed in several well-established cases including CrkL, Src, and SHP-1/2 (Lu et al., 2001; Rosen et al., 1995; Sicheri et alo, 1997; Xu et alo, 1997; Zhang et al., 2003). Each of these examples involves an $\mathrm{SH} 2$ domain interacting with a phosphotyrosine. We propose a Src-like model for PTEN, in which a cluster of pSer/pThr drives an apparently long distance intramolecular binding interaction to deactivate the enzyme. How 4p-PTEN may be reactivated in vivo remains an important question. There are presumably cellular phosphatase enzymes that can dephosphorylate phospho-PTEN or ligands which can bind allosterically to phospho-PTEN that may promote conformational opening. In contrast to prior suggestions based on trans experiments, we see no evidence of the autodephosphorylation of 4p-PTEN (Zhang et alı, 2012). As we are reliant on western blots with an antibody that may recognize states of depleted phosphorylation of the tail, partial autodephosphorylation cannot be completely ruled out by our experiments. However, the stability of the closed conformation of 4p-PTEN over the dozens of hours of expressed protein ligation points to resistance of the tail phosphates to PTEN enzymatic removal. Because of the structural concealment of the 380-5 phosphoSer/Thr residues in the closed 4p-PTEN conformation, such autodephosphorylation may be especially disfavored.

There are several biomedical implications of the findings here. Altered protein-protein interactions or efficiency of ubiquitylation may be influenced by phosphorylation-mediated conformational closure of phospho-PTEN. While PTEN is a tumor suppressor and can be mutated in cancer, it is often wild type but expressed at low levels. Direct stimulation of cellular phospho-PTEN by pharmacologic agents could prove to be effective as an anti-cancer therapy. A related approach has been explored to activate the tumor suppressor p53 (Foster et al., 1999) and the apoptotic protein procaspase (Gray et al., 2010). It may be possible to find small molecules that bind specifically to the phospho-tail and prevent its intramolecular engagement with the $\mathrm{C} 2$ domain or bind somewhere on the PTEN body and stabilize the open PTEN conformation allosterically. The soluble $\mathrm{PIP}_{3}$ substrate dephosphorylation assay with $4 p$-PTEN should provide a means for screening for such activators. Alternatively, inhibitors of CK2 and/or GSK3 $\beta$ protein kinase, the enzymes responsible for PTEN C-tail phosphorylation, may be effective in targeting $\mathrm{PIP}_{3} /$ Akt-driven tumors.

This investigation also highlights the use of intein-mediated protein thioester formation in an insect cell expression system for investigation of post-translational modifications. Multi-site phosphorylation has been elegantly studied in the TGF $\beta$ signaling axis using protein semisynthesis previously (Huse et al., 2001; Wu et al., 2001), but these experiments did not require insect cell expression of an inteinfusion protein. Our efforts here suggest that baculovirus expression systems are an attractive option, rather than a last resort, for expressed protein ligation with challenging eukaryotic proteins.

\section{Materials and methods}

\section{Reagents}

All lipids were from Avanti Polar Lipids (Alabaster, AL). Antibodies were from Santa Cruz Biotechnology (Dallas, TX) (SC-6818) and Novus Biological (Littleton, CO) (NBP1-4136 and NBP1-44,412). MESNA was from Sigma (St. Louis, MO). All Fmoc-amino acids were from EMD (Billerica, MA).

\section{Peptide synthesis}

All peptides were synthesized on a PS3 peptide synthesizer from Protein Technologies (Tuscon, AZ) or by hand using Fmoc based standard solid phase peptide synthesis. 


\section{Generation and purification of semisynthetic PTEN}

PTEN C-terminally truncated at residue 378 was first subcloned into the pTXB1 vector from NEB which contains the GyrA intein from the organism Mycobacterium xenopi. Tyr 379 was mutated to a Cys to facilitate the intein mediated cleavage reaction. The PTEN-intein-cbd DNA sequence was then subcloned into the pFastBac1 baculovirus entry vector and the subsequent baculovirus was generated. The PTEN-intein-cbd fusion protein was expressed in HighFive insect cells.

The fusion protein in $40 \mathrm{ml}$ of cell lysate from 1 I of cell culture was first passed over a $10 \mathrm{ml}$ bed of fibrous cellulose (Whatman) to remove viral chitinase, then bound to a $6 \mathrm{ml}$ bed of chitin beads from NEB in a gravity flow chromatography column from Bio-Rad ( $2.5 \mathrm{~cm}$ diameter). The chitin bead column with fusion protein bound was then washed with $250 \mathrm{ml}$ of washing buffer $(50 \mathrm{mM} \mathrm{HEPES} \mathrm{pH} \mathrm{7.6,}$ $250 \mathrm{mM} \mathrm{NaCl}, 0.1 \%$ Triton X-100). C-terminally truncated PTEN (t-PTEN) was generated by DTT cleavage of the fusion protein, producing t-PTEN at yields of $\sim 8-10 \mathrm{mg}$ per liter of cell culture. Full length semisynthetic PTEN was then generated on the chitin column by adding $400 \mathrm{mM}$ MESNA and $2 \mathrm{mM}$ of C-terminal peptide buffered with $3 \mathrm{ml}$ of $50 \mathrm{mM} \mathrm{HEPES} \mathrm{(pH} \mathrm{7.2),} 150 \mathrm{mM} \mathrm{NaCl}$. Based on the post ligation yield of PTEN protein it is estimated that there is a maximum PTEN-thioester concentration of $\sim 80 \mu \mathrm{M}$ in the ligation reaction. The ligation reactions were carried out for $48-72 \mathrm{hr}$ at room temperature and monitored by SDS PAGE. Upon completion of the ligation reaction the ligation mixture was eluted from the chromatography column with $15 \mathrm{ml}$ of dialysis buffer $(50 \mathrm{mM}$ Tris $\mathrm{pH} 8.0$, $150 \mathrm{mM} \mathrm{NaCl}, 10 \mathrm{mM} \mathrm{DTT}$ ) and subsequently dialyzed into 4 I of dialysis buffer for a period of $48 \mathrm{hr}$ with multiple buffer exchanges in a dialysis cassette (Slidealyzer) with a $12 \mathrm{~K} \mathrm{MWCO}$ in order to remove excess unreacted peptide. Proteins were then concentrated following dialysis to $>5 \mathrm{mg} / \mathrm{ml}(>100 \mu \mathrm{M})$. We estimate that there is $<10 \mu \mathrm{M}$ of residual unligated peptides at this stage. Due to large dilutions (>1000-fold) of the semisynthetic enzyme for enzymatic and other biochemical assays, small amounts of residual contaminating peptide remaining after dialysis would not be expected to interfere with any assays. Semisynthetic PTEN proteins produced in this way yields 8-10 mg of protein per liter of insect cell culture with the desired modifications on the C-terminus at purities of $>90 \%$ based on Coomassie stained SDSPAGE. The semisynthetic protein was further purified for SAXS and soluble substrate assays by anion exchange chromatography (monoQ) using an AKTA FPLC from GE Healthcare. Proteins were purified with a gradient of 0-50\% Buffer B over $250 \mathrm{ml}$ at a flow rate of $1.0 \mathrm{ml} / \mathrm{min}$ (Buffer A: $50 \mathrm{mM}$ Tris pH 8.0, 10 mM DTT; Buffer B: 50 mM Tris pH 8.0, 1.0 M NaCl, 10 mM DTT). After FPLC purification by anion exchange chromatography and concentration the final yield of semisynthetic PTEN protein was 2-3 mg per liter of cell culture with estimated purity $>95 \%$ by coomassie stained SDS-PAGE. Size-exclusion chromatography was carried out with a Superdex 200 column in the following buffer: $50 \mathrm{mM}$ Tris pH8.0, $150 \mathrm{mM} \mathrm{NaCl}, 10 \mathrm{mM}$ DTT.

\section{Generation of radiolabed PIP $_{3}$}

Radiolabeled $\mathrm{PIP}_{3}$ was generated as previously described (McConnache et al., 2003). Briefly, PIP 3 labeled with ${ }^{32} \mathrm{P}_{\mathrm{i}}$ at the three position of the inositol ring was generated by incubating $\mathrm{PI} 3 \mathrm{~K}$ (Echleon) and PIP $_{2}$ :PS (1:1) vesicles in the presence of $250 \mathrm{mCi}{ }^{32} \mathrm{P}-\mathrm{ATP}, 1 \mathrm{mM}$ ATP and $2.5 \mathrm{mM} \mathrm{MgCl}$ in PI3K assay buffer (25 mM HEPES pH 7.6, $120 \mathrm{mM} \mathrm{NaCl}$ and $1 \mathrm{mM}$ EGTA). After a Bligh-Dyer extraction of $\mathrm{PIP}_{3}$, thin-layer chromatography (TLC) showed radiolabeled $\mathrm{PIP}_{3}$ to be the major product (Figure 2-figure supplement 3). TLC solvent conditions: $\left(\mathrm{CHCl}_{3}\right.$ :Acetone:MeOH;Acetic Acid: $\left.\mathrm{H}_{2} \mathrm{O}\right) ;(70: 20: 50: 20: 20)$.

\section{Vesicle based phosphatase assays}

Lipid phosphatase assays were modified from those already described in the literature (McConnachie et al., 2003). ${ }^{32} \mathrm{P}$ radiolabeled $\mathrm{PIP}_{3}$ was incorporated into vesicles containing unlabeled $\mathrm{PIP}_{3}$, phosphatidylcholine (PC), and/or phosphatidylserine (PS) and/or $\mathrm{PIP}_{2}$ by sonication of dried lipids hydrated in the presence of PTEN assay buffer (50 mM Tris pH 8.0, $150 \mathrm{mM} \mathrm{NaCl}, 10 \mathrm{mM}$ DTT, $1 \mathrm{mM}$ EGTA). Lipids were sonicated in $100 \mu$ volumes in glass test tubes at room temperature until the solution clarified. Vesicles made in this way are 30-50 nm in diameter. Vesicles were used in assays within $15 \mathrm{~min}$ of being made. In assay reactions, the ratio of the number of vesicles to the number of PTEN molecules was maintained at $4: 1$ or greater. Ovalbumin $(0.05 \mathrm{mg} / \mathrm{ml})$ was used to stabilize the PTEN protein in the assays. $25 \mu \mathrm{l}$ reactions were initiated by the addition of vesicle substrate and incubated at $30^{\circ} \mathrm{C}$ for $3 \mathrm{~min}$. The reaction was quenched with $3 \mathrm{M}$ perchloric acid. Hydrolyzed ${ }^{32} \mathrm{P}_{i}$ was then separated from ${ }^{32} \mathrm{P}-\mathrm{PIP}_{3}$ by a Bligh-Dyer extraction. The aqueous phase was then treated with $1 \%$ ammonium 
molybdate and the resulting phosphate-molybdate complex was extracted with toluene:isobutanol $(1: 1)$. This organic phase was then counted using a Beckman scintillation counter.

\section{Interfacial kinetic analysis}

Analysis of the kinetic parameters of the semisynthetic PTEN proteins were determined in accordance with the procedures pioneered by Dennis and coworkers and previously performed for recombinant PTEN produced in E. coli (Deems et al., 1975; Hendrickson and Dennis, 1984; McConnachie et al., 2003). With this type of analysis, the initial velocity of an interfacial enzyme follows the equation below:

$$
\mathrm{V}_{0}=\left(\mathrm{V}_{\max }{ }^{*} \mathrm{X}_{\mathrm{s}}{ }^{*}\left[\mathrm{~S}_{0}\right]\right) /\left(\mathrm{i} K_{\mathrm{m}}{ }^{*} \mathrm{~K}_{\mathrm{s}}+\mathrm{i} K_{\mathrm{m}}{ }^{*}\left[\mathrm{~S}_{0}\right]+\mathrm{X}_{\mathrm{s}}{ }^{*}\left[\mathrm{~S}_{0}\right]\right)
$$

Two types of experiments were performed, bulk dilution (BD) and surface dilution (SD). In BD experiments, the surface concentration of $\mathrm{PIP}_{3}$ was held constant and bulk concentration of $\mathrm{PIP}_{3}$ was varied by varying the concentration of $\mathrm{PIP}_{3}$ and the carrier lipid $\mathrm{PC}$ proportionately. In SD experiments, the bulk concentration of $\mathrm{PIP}_{3}$ was held constant and the surface concentration was varied by varying the amount of PC. In both types of experiments rectangular hyperbolas were obtained with apparent $V_{\max }$ and apparent $K_{m}$ values. Apparent $V_{\max }$ and apparent $K_{m}$ values were then fit to the equations below to determine the kinetic variables for each PTEN protein.

$$
\begin{aligned}
& \mathrm{i} k_{\mathrm{m}}=\left(\mathrm{V}_{\max } \mathrm{SD} / \mathrm{V}_{\max } \mathrm{BD}-1\right) \mathrm{X}_{\mathrm{s}} \\
& \mathrm{K}_{\mathrm{s}}=\mathrm{K}_{\mathrm{m}} \mathrm{BD}\left(\mathrm{X}_{\mathrm{s}} / \mathrm{i}_{\mathrm{m}}+1\right) \\
& \mathrm{k}_{\text {cat }}=\mathrm{V}_{\max } \mathrm{SD}^{*}\left[\mathrm{E}_{\mathrm{T}}\right]
\end{aligned}
$$

\section{Vesicle pulldowns}

Large Multilamillar Vesicles (LMVs) containing various amounts of PC, PS and/or PIP 2 were generated by vigorously vortexing dried lipids that were hydrated in the presence of PTEN buffer for $5 \mathrm{~min}$ in $1 \mathrm{ml}$ volumes. The LMVs were then incubated with different forms of PTEN protein for $30 \mathrm{~min}$ at $25^{\circ} \mathrm{C}$. The vesicles and bound protein in $50 \mu \mathrm{l}$ volumes were then pelleted at 180,000 $\mathrm{g}$ using a Beckman ultracentrifuge for $2 \mathrm{hr}$. The supernatant was removed from the vesicle pellet, the pellet washed with buffer, then boiled in $10 \%$ SDS loading dye and run on SDS-PAGE. The amount of PTEN protein that bound to the LMVs was then visualized by western blot using an anti-PTEN antibody from Santa Cruz Biotechnologies (SC-6818). The amount bound was quantified using Carestream Media image quantification software. For tail peptide competition assays the amount of tail peptide used was $10 \mu \mathrm{M}$.

\section{Trypsin digests}

$2 \mu \mathrm{g}$ of semisynthetic PTEN in $20 \mu \mathrm{l}$ reactions volumes was digested with varying amounts of trypsin (Promega, V511A) for $10 \mathrm{~min}$ at $37^{\circ} \mathrm{C}$ in PTEN assay buffer. Reactions then were quenched with SDS loading dye and run on SDS-PAGE. The digestion fragments were visualized by Colloidal Blue stain from Invitrogen (LC6025) or by western blot (antibodies SC-6818 or NBP1-44,412).

\section{Sequencing of trypsin digest fragments}

Trypsin digestion products were run on a $10 \%$ SDS-PAGE gel and transferred to a PVDF membrane. The membrane was then stained with Coomassie stain. The bands of interest were cut out of the membrane and analyzed by N-terminal Edman degradation sequencing at the JHMI Synthesis and Sequencing Facility.

\section{Phosphatase sensitivity assay}

$50 \mathrm{ng}$ of semisynthetic phosphorylated PTEN and its mutants were dephosphorylated in the presence of $1 \mu \mathrm{M}$ alkaline phosphatase from NEB (CIP) for varying periods of time in phosphatase assay buffer (50 mM Tris $\mathrm{pH} 8.0,20 \mathrm{mM} \mathrm{NaCl}, 25 \mu \mathrm{M} \mathrm{MgCl}_{2}$ and $10 \mathrm{mM} \mathrm{DTT}$ ) at room temperature in $20 \mu \mathrm{l}$. Dephosphorylation of PTEN was monitored by western blot with an antibody to the phospho-tail cluster (NBP1-4136). The fraction of phospho-PTEN remaining was determined using Carestream Media image quantification software.

\section{Soluble substrate activity assay}

PTEN activity to a water soluble substrate $\left(\mathrm{diC} \mathrm{PIP}_{3}\right)$ was determined by measuring the release of inorganic phosphate with a malachite green (Van Veldhoven and Mannaert, 1987) detection kit from $\mathrm{R}$ and $\mathrm{D}$ Biosystems. $25 \mu \mathrm{l}$ reactions were allowed to proceed for $5-10$ minutes at $30^{\circ} \mathrm{C}$ in assay buffer 
(50 mM Tris pH 8.0, $10 \mathrm{mM} \mathrm{BME)} \mathrm{before} \mathrm{being} \mathrm{quenched} \mathrm{by} \mathrm{malachite} \mathrm{green} \mathrm{reagent.} \mathrm{Amounts} \mathrm{of} \mathrm{PTEN}$ used per data point ranged from 0.5 to $20 \mu \mathrm{g}$. Reactions were shown to be linear with respect to time and enzyme concentration in the ranges used. For in trans peptide inhibition assays, the amount of tail peptide used (quantified by amino acid analysis) is indicated in the figure legend. It is unlikely that any peptide ligated to t-PTEN given the low concentrations of peptide used, short reaction times and the low reactivity of the DTT-thioester toward native chemical ligation. In fact, with the vesicle pulldowns that used $10 \mu \mathrm{M}$ phosphopeptide and t-PTEN, there is no evidence of ligation observed by western blot.

\section{Small-angle X-ray scattering (SAXS)}

SAXS experiments were performed at Brookhaven National Laboratories at the National Synchrotron Light Source (NSLS), beamline X9 using a MarCCD detector, located $3.4 \mathrm{~m}$ from the sample. Data for each protein sample was collected in triplicate. All samples were in PTEN assay buffer. $20 \mu \mathrm{l}$ of each sample was continuously passed through a capillary tube exposed to a $400 \times 200 \mu \mathrm{m}$ X-ray beam and data recorded for 30s. Normalization for beamline intensity, buffer subtraction and merging of data were carried out using proc.py software developed by the beamline staff (Allaire and Yang, 2011).

SAXS data analysis was carried out using software from the ATSAS program suite. The radius of gyration $\left(R_{g}\right)$ was calculated using a Guinier approximation with the program PRIMUS (Konarev et al., 2003). The pair distribution function $P(r)$ and the maximum particle dimension $\left(D_{\max }\right)$ were determined using GNOM (Svergun et al., 1992). Ten ab initio models were generated for each protein using DAMMIN, then averaged using DAMAVER (Volkov and Svergun, 2003; Putnam et al., 2007; Franke and Svergun, 2009). The resulting molecular envelopes were fit with the tailless crystal structure in PyMOL. Figures of the models were made using PyMOL.

\section{Acknowledgements}

We thank T Woolf, MK Tarrant, and M Allaire for assistance and helpful advice. We are grateful to the NIH for support.

\section{Additional information}

Competing interests

PC: Reviewing editor, eLife. The other authors declare that no competing interests exist.

Funding

\begin{tabular}{lll} 
Funder & Grant reference number & Author \\
\hline National Institutes of Health & & $\begin{array}{l}\text { Philip Cole, } \\
\text { Peter Devreotes }\end{array}$ \\
\hline National Institutes of Health & 4R37CA043460 & $\begin{array}{l}\text { Sandra B Gabelli, } \\
\text { L Mario Amzel }\end{array}$
\end{tabular}

The funders had no role in study design, data collection and

interpretation, or the decision to submit the work for publication.

Author contributions

DB, MR, SBG, Conception and design, Acquisition of data, Analysis and interpretation of data, Drafting or revising the article; BT-S, Acquisition of data, Analysis and interpretation of data, Drafting or revising the article; SCS, Acquisition of data, Drafting or revising the article, Contributed unpublished essential data or reagents; DR, LMA, PD, PC, Conception and design, Analysis and interpretation of data, Drafting or revising the article

\section{References}

Al-Khouri AM, Ma Y, Togo SH, Williams S, Mustelin T. 2005. Cooperative phosphorylation of the tumor suppressor phosphatase and tensin homologue (PTEN) by casein kinases and glycogen synthase kinase 3beta. J Biol Chem 280:35195-202. doi: 10.1074/jbc.M503045200.

Allaire M, Yang L. 2011. Biomolecular solution X-ray scattering at the National Synchroton Light Source. J Synchrotron Radiat 18:41-4. doi: 10.1107/S0909049510036022. 
Campbell RB, Liu F, Ross AH. 2003. Allosteric activation of PTEN phosphatase by phosphatidylinositol 4,5-bisphosphate. J Biol Chem 278:33617-20. doi: 10.1074/jbc.C300296200.

Chagpar R, Links PH, Pastor MC, Furber LA, Hawrysh AD, Chamberlain MD, et al. 2009. Direct positive regulation of PTEN by the p85 subunit of phophatidylinsoitol 3-Kinase. Proc Natl Acad Sci USA 107:5471-6. doi: 10.1073/pnas.0908899107.

Chung WK, Hou Y, Freed A, Holstein M, Makhatadze GI, Cramer SM. 1989. Investigation of protein binding affinity and preferred orientations in ion exchange systems using homologous protein library. Anal Chem 61:1742-50. doi: 10.1002/bit.22100.

Cordier F, Chaffotte A, Terrien E, Prehaud C, Theillet FX, Delepierre M, et al. 2012. Ordered phosphorylation events in two independent cascades of the PTEN C-tail revealed by NMR. J Am Chem Soc 134:20533-43. doi: 10.1021/ja310214g.

Deems RA, Eaton BR, Dennis EA. 1975. Kinetic analysis of phospholipase A2 activity toward mixed micelles and its implications for the study of lipolytic enzymes. J Biol Chem 250:9013-20.

Denning G, Jean-Joseph B, Prince C, Durden DL, Vogt PK. 2007. A short N-terminal sequence of PTEN controls cytoplasmic localization and is required for suppression of cell growth. Oncogene 26:3930-40. doi: 10.1038/sj.onc.1210175.

Di Cristofano A, Pesce B, Cordon-Cardo C, Pandolfi PP. 1999. Pten is essential for embryonic development and tumour suppression. Nat Genet 19:348-55. doi: 10.1038/1235.

Foster BA, Coffey HA, Morin MJ, Rastinejad F. 1999. Pharmacological rescue of mutant p53 conformation and function. Science 286:2507-10. doi: 10.1126/science.286.5449.2507.

Franke D, Svergun DI. 2009. DAMMIF, a program for rapid ab-initio shape determination in small-angle scattering. J Appl Cryst 42:342-6. doi: 10.1107/S0021889809000338.

Gray DC, Mahrus S, Wells JA. 2010. Activation of specific apoptotic caspases with an engineered small-moleculeactivated protease. Cell 142:637-46. doi: 10.1016/j.cell.2010.07.014.

Hendrickson HS, Dennis EA. 1984. Analysis of the kinetics of phospholipid activation of cobra venom phospholipase A2. J Biol Chem 259:5740-4.

Hollander MC, Blumenthal GM, Dennis PA. 2011. PTEN loss in the continuum of common cancers, rare syndromes and mouse models. Nature Rev Cancer 11:289-301. doi: 10.1038/nrc3037.

Hou Y, Hansen TB, Staby A, Cramer SM. 2010. Effects of urea induced protein conformational changes on ion exchange chromatographic behavior. J Chromatogr A 1217:7393-400. doi: 10.1016/j.chroma.2010.09.052.

Huang J, Yan J, Zhang J, Zhu S, Wang Y, Shi T, et al. 2012. SUMO1 modification of PTEN regulates tumorigenesis by controlling its association with the plasma membrane. Nat Commun 3:911. doi: 10.1038/ncomms1919.

Huse M, Muir TW, Xu L, Chen YG, Kuriyan J, Massague J. 2001. The TGF beta receptor activation process: an inhibitor-to substrate-binding switch. Mol Cell 8:671-82. doi: 10.1016/S1097-2765(01)00332-X.

lijima M, Devreotes P. 2002. Tumor suppressor PTEN mediates sensing of chemoattractant gradients. Cell 109:599-610. doi: 10.1016/S0092-8674(02)00745-6.

lijima M, Huang YE, Luo HR, Vazquez F, Devreotes PN. 2004. Novel mechanism of PTEN regulation by its phosphatidylinositol 4,5-bisphosphate binding motif is critical for chemotaxis. J Biol Chem 279:16606-13. doi: 10.1074/jbc.M312098200.

Jacques DA, Trewhella J. 2012. Small-angle scattering for structural biology-expanding the frontier while avoiding the pitfalls. Protein Sci 19:642-57. doi: 10.1002/pro.351.

Konarev PV, Volkov VV, Sokolova AV, Koch MHJ, Svergun DI. 2003. PRIMUS-a Windows-PC based system for small-angle scattering data analysis. J Appl Cryst 36:1277-82. doi: 10.1107/S0021889803012779.

Lee JO, Yang H, Georgescu MM, Di Cristofano A, Maehama T, Shi Y, et al. 1999. Crystal structure of the PTEN tumor suppressor: Implications for its phosphoinositide phosphatase activity and membrane association. Cell 99:323-34. doi: 10.1016/S0092-8674(00)81663-3.

Li J, Yen C, Liaw D, Podsypanina K, Bose S, Wang SI, et al. 1997. PTEN, a putative protein tyrosine phosphatase gene mutated in human brain, breast, and prostate cancer. Science 275:1943-7. doi: 10.1126/ science.275.5308.1943.

Lu W, Gong D, Bar-Sagi D, Cole PA. 2001. Site-specific incorporation of a phosphotyrosine mimetic reveals a role for tyrosine phosphorylation of SHP-2 in cell signaling. Mol Cell 8:759-69. doi: 10.1016/ S1097-2765(01)00369-0.

Maehama T, Dixon JE. 1998. The tumor suppressor, PTEN/MMAC1, dephosphorylates the lipid second messenger, phosphatidylinositol 3,4,5-trisphosphate. J Biol Chem 273:13375-8. doi: 10.1074/jbc.273.22.13375

McConnachie G, Pass I, Walker SM, Downes CP. 2003. Interfacial kinetic analysis of the tumour suppressor phosphatase, PTEN: evidence for activation by anionic phospholipids. Biochem J 371:947-55. doi: 10.1042/BJ20021848.

Meng F, Henson R, Wehbe-Janek H, Ghoshal K, Jacob ST, Patel T. 2007. MicroRNA-21 regulates expression of the PTEN tumor suppressor gene in human hepatocellular cancer. Gastroenterology 133:647-58. doi: 10.1053/j.gastro.2007.05.022.

Miller SJ, Lou DY, Seldin DC, Lane WS, Neel BG. 2002. Direct identification of PTEN phosphorylation sites. FEBS Lett 528:145-53. doi: 10.1016/S0014-5793(02)03274-X.

Muir TW, Sondhi D, Cole PA. 1998. Expressed protein ligation: a general method for protein engineering. Proc Natl Acad Sci USA 95:6705-10. doi: 10.1073/pnas.95.12.6705.

Myers MP, Pass I, Batty IH, Van der Kayy J, Stolarov JP, Hemmings BA, et al. 1998. The lipid phosphatase activity of PTEN is critical for its tumor supressor function. Proc Natl Acad Sci USA 95:13513-8. doi: 10.1073/pnas.95.23.13513.

Nelen MR, van Staveren WC, Peeters EA, Hassel MB, Gorlin RJ, Hamm H, et al. 1997. Germline mutations in the PTEN/MMAC1 gene in patients with Cowden disease. Hum Mol Genet 6:1383-7. doi: 10.1093/hmg/6.8.1383. 
Odriozola L, Singh G, Hoang T, Chan AM. 2007. Regulation of PTEN activity by its carboxyl-terminal autoinhibitory domain. J Biol Chem 282:23306-15. doi: 10.1074/jbc.M611240200.

Okumura K, Mendoza M, Bachoo RM, DePinho RA, Cavenee WK, Furnari FB. 2006. PCAF modulates PTEN activity. J Biol Chem 281:26562-8. doi: 10.1074/jbc.M605391200.

Podsypanina K, Wllenson LH, Nemes A, Gu J, Tamura M, Yamada KM, et al. 1999. Mutation of Pten/Mmac1 in mice causes neoplasia in multiple organ systems. Proc Natl Acad Sci USA 96:1563-8. doi: 10.1073/pnas.96.4.1563.

Pradhan S, Bacolla A, Wells RD, Roberts RJ. 1999. Recombinant human DNA (cytosine-5) methyltransferase. I. Expression, purification and comparison of de novo maintenance methylation. J Biol Chem 274:33002-10. doi: 10.1074/jbc.274.46.33002.

Putnam CD, Hammel M, Hura GL, Tainer JA. 2007. X-ray solution scattering (SAXS) combined with crystallography and computation: defining accurate macromolecular structures, conformations and assemblies in solution. Q Rev Biophys 40:191-285. doi: 10.1017/S0033583507004635.

Rahdar M, Inoue T, Meyer T, Zhang J, Vazquez F, Devreotes PN. 2009. A phosphorylation-dependent intramolecular interaction regulates the membrane association and activity of the tumor suppressor PTEN. Proc Natl Acad Sci USA 106:480-5. doi: 10.1073/pnas.0811212106.

Rosen MK, Yamazaki T, Gish GD, Kay CM, Pawson T, Kay LE. 1995. Direct demonstration of an intramolecular SH2-phosphotyrosine interaction in the Crk protein. Nature 374:477-9. doi: 10.1038/374477a0.

Salvesen HB, MacDonald N, Ryan A, Jacobs IJ, Lynch ED, Akslen LA, et al. 2001. PTEN methylation is associated with advanced stage and microsatellite instability in endometrial carcinoma. Int J Cancer 91:22-6. doi: 10.1002/1097-0215(20010101)91:1<22::AID-IJC1002>3.0.CO;2-S.

Schwarzer D, Cole PA. 2005. Protein semisynthesis and expressed protein ligation: chasing a protein's tail. Curr Opin Chem Biol 9:561-9. doi: 10.1016/j.cbpa.2005.09.018.

Shenoy S, Shekhar P, Heinrich F, Daou MC, Gericke A, Ross AH, et al. 2012. Membrane association of the PTEN tumor suppressor: molecular details of the protein-membrane complex from SPR binding studies and neutron reflection. PLOS ONE 7:e32591. doi: 10.1371/journal.pone.0032591.

Sicheri F, Moarefi I, Kuriyan J. 1997. Crystal structure of the Src family tyrosine kinase Hck. Nature 385:602-9. doi: 10.1038/385602a0.

Song MS, Salmena L, Pandolfi PP. 2012. The functions and regulation of the PTEN tumour suppressor. Nat Rev Mol Cell Bio 13:283-96. doi: 10.1038/nrm3330.

Steck PA, Pershouse MA, Jasser SA, Yung WK, Lin H, Ligon AH, et al. 1997. Identification of a candidate tumour suppressor gene, MMAC1, at chromosome 10q23.3 that is mutated in multiple advanced cancers. Nat Genet 15:356-62. doi: 10.1038/ng0497-356.

Sumitomo M, Iwase A, Zheng R, Navarro D, Kaminetzky D, Shen R, et al. 2004. Synergy in tumor suppression by direct interaction of neutral endopeptidase with PTEN. Cancer Cell 5:67-78. doi: 10.1016/S1535-6108(03)00331-3.

Sun H, Lesche R, Li DM, Liliental J, Zhang H, Gao J, et al. 1999. PTEN modulates cell cycle progression and cell survival by regulating phosphatidylinositol 3,4,5,-trisphosphate and Akt/protein kinase B signaling pathway. Proc Natl Acad Sci USA 96:6199-204. doi: 10.1073/pnas.96.11.6199.

Suzuki A, de la Pompa JL, Stambolic V, Elia AJ, Sasaki T, del Barco Barrantes I, et al. 1998. High cancer susceptibility and embryonic lethality associated with mutation of the PTEN tumor suppressor gene in mice. Curr Biol 8:1169-78. doi: 10.1016/S0960-9822(07)00488-5.

Svergun DI. 1992. Determination of the regularization parameter in indirect transform methods using perceptual criteria. J Appl Crystallogr 26:495-503. doi: 10.1107/S0021889892001663.

Takahashi Y, Morales FC, Kreimann EL, Georgescu MM. 2006. PTEN tumor suppressor associates with NHERF proteins to attenuate PDGF receptor signaling. EMBO J 25:910-20. doi: 10.1038/sj.emboj.7600979.

Torres J, Pulido R. 2001. The tumor suppressor PTEN is phosphorylated by the protein kinase CK2 at its C terminus. implications for PTEN stability to proteasome-mediated degradation. J Biol Chem 276:993-8. doi: $10.1074 / j b c . M 009134200$.

Van Diepen MT, Parsons M, Downes CP, Leslie NR, Hindges R, Eickholt BJ. 2009. MyosinV controls PTEN function and neuronal cell size. Nature Cell Biol 11:1191-6. doi: 10.1038/ncb1961.

Van Veldhoven PP, Mannaert GP. 1987. Inorganic and organic phosphate measurements in the nanomolar range. Anal Biochem 161:45-8. doi: 10.1016/0003-2697(87)90649-X.

Vazquez F, Grossman SR, Takahashi Y, Rokas MV, Nakamura N, Sellers WR. 2001. Phosphorylation of the PTEN tail acts as an inhibitory switch by preventing its recruitment into a protein complex. $\mathrm{J}$ Biol Chem 276:48627-30. doi: 10.1074/jbc.C100556200.

Vazquez F, Matsuoka S, Sellers WR, Yanagida T, Ueda M, Devreotes PN. 2006. Tumor suppressor PTEN acts through dynamic interaction with the plasma membrane. Proc Natl Acad Sci USA 13:3633-8. doi: 10.1073/ pnas.0510570103.

Vazquez F, Ramaswamy S, Nakamura N, Sellers WR. 2000. Phosphorylation of the PTEN tail regulates protein stability and function. Mol Cell Biol 20:5010-8. doi: 10.1128/MCB.20.14.5010-5018.2000.

Vila-Perelló M, Muir TW. 2010. Biological applications of protein splicing. Cell 143:191-200. doi: 10.1016/j. cell.2010.09.031.

Volkov VV, Svergun DI. 2003. Uniqueness of ab-initio shape determination in small-angle scattering. J App/ Cryst 36:860-4. doi: 10.1107/S0021889803000268.

Walker SM, Leslie NR, Perera NM, Batty IH, Downes CP. 2004. The tumour-suppressor function of PTEN requires an N-terminal lipid-binding motif. Biochem J 379:301-7. doi: 10.1042/BJ20031839.

Wang D, Esselman WJ, Cole PA. 2002. Substrate conformational restriction and CD45-catalyzed dephosphorylation of tail tyrosine-phosphorylated Src protein. J Biol Chem 277:40428-33. doi: 10.1074/jbc.M206467200. 
Wang X, Trotman LC, Koppie T, Alimonti A, Chen Z, Gao Z, et al. 2007. NEDD4-1 is a proto-oncogenic ubiquitin ligase for PTEN. Cell 128:129-39. doi: 10.1016/j.cell.2006.11.039.

Wu X, Hepner K, Castelino-Prabhu S, Do D, Kaye MB, Yuan XJ, et al. 2000. Evidence for regulation of the PTEN tumor suppressor by a membrane-localized multi-PDZ domain containing scaffold protein MAGI-2. Proc Natl Acad Sci USA 97:4233-8. doi: 10.1073/pnas.97.8.4233.

Wu JW, Hu M, Chai J, Seoane J, Huse M, Li C, et al. 2001. Crystal structure of a phosphorylated Smad2. Recognition of phosphoserine by the $\mathrm{MH} 2$ domain and insights on Smad function in TGF-beta signaling. Mol Cell 8:1277-89. doi: 10.1016/S1097-2765(01)00421-X.

Xu W, Harrison SC, Eck MJ. 1997. Three-dimensional structure of the tyrosine kinase c-Src. Nature 385:595-602. doi: 10.1038/385595a0.

Zhang Z, Shen K, Lu W, Cole PA. 2003. The role of c-terminal tyrosine phosphorylation in the regulation of SHP-1 explored via expressed protein ligation. J Biol Chem 278:4668-74. doi: 10.1074/jbc.M210028200.

Zhang XC, Piccini A, Myers MP, Van Aelst L, Tonks NK. 2012. Functional analysis of the protein phosphatase activity of PTEN. Biochem J 444:457-64. doi: 10.1042/BJ20120098. 